\title{
Dynamic prediction-based relocation policies in one-way station-based carsharing systems with complete journey reservations
}

\author{
Martin Repoux ${ }^{\mathrm{a}}$, Mor Kaspi ${ }^{\mathrm{b}}$, Burak Boyaci ${ }^{\mathrm{c}}$, Nikolas Geroliminis ${ }^{\mathrm{a}, *}$ \\ ${ }^{a}$ Urban Transport Systems Laboratory \\ School of Architecture, Civil and Environmental Engineering \\ École Polytechnique Fédérale de Lausanne (EPFL) \\ Station 18, CH-1015 Lausanne, Switzerland \\ ${ }^{b}$ Analytics for Urban Transportation and Operations Laboratory (AUTO) \\ Department of Industrial Engineering, Tel Aviv University \\ Tel Aviv, Israel \\ ${ }^{c}$ Centre for Transport and Logistics (CENTRAL) \\ Lancaster University Management School \\ Lancaster, LA1 4YX, UK
}

\begin{abstract}
In this paper, we study the operations of a one-way station-based carsharing system implementing a complete journey reservation policy. We consider the percentage of served demand as a primary performance measure and analyze the effect of several dynamic staff-based relocation policies. Specifically, we introduce a new proactive relocation policy based on Markov chain dynamics that utilizes reservation information to better predict the future states of the stations. This policy is compared to a state-of-the art staff-based relocation policy and a centralistic relocation model assuming full knowledge of the demand. Numerical results from a real-world implementation and a simulation analysis demonstrate the positive impact of dynamic relocations and highlight the improvement in performance obtained with the proposed proactive relocation policy.
\end{abstract}

Keywords: Carsharing, Simulation, Markov chain, Prediction, Operations

\section{Introduction}

Human mobility in modern cities is a highly complex dynamical system with many modes of transport that interact and compete for urban space. New modes of transportation and ways of moving, among them shared-mobility systems, create additional opportunities to decrease car ownership and congestion but also additional challenges for an efficient management of these fleets. Carsharing is a type of shared-mobility system consisting of a fleet of vehicles distributed in an urban area that can be accessed on-street and rented for short durations by pre-registered users. Usage cost is in general a function of both renting duration and distance traveled. Such a system aims at providing a level of service similar to private vehicles while mutualizing the transportation resources. This has several benefits both at user and society scale. It spreads vehicle ownership fixed costs, such as purchase, insurance and maintenance, over multiple households (Katzev (2003)). By doing so, it provides an attractive mobility alternative to a greater share of the population (Duncan (2011); Litman (2000)) and has potential to overall reduce greenhouse gas emissions due to automotive transportation (Firnkorn and Müller (2011); Martin and Shaheen (2011)), mitigate congestion and free up parking spaces (Crane et al. (2012)). Therefore, many studies (Schuster et al. (2005); Costain et al. (2012)) have analyzed carsharing growth and shed light on the factors favoring it as part of a sustainable transportation future and making it a viable alternative to private car. Recent trends, statistics, as well as global perspectives related to carsharing and its future can be found in Shaheen and Cohen (2013); Shaheen et al. (2018).

\footnotetext{
${ }^{*}$ Corresponding author

Email addresses: martin.repoux@epfl.ch (Martin Repoux), morkaspi@tauex.tau.ac.il (Mor Kaspi), b.boyaci@lancaster.ac.uk (Burak Boyac1), nikolas.geroliminis@epfl.ch (Nikolas Geroliminis)
} 
Existing carsharing systems can be classified into a variety of types based on the following main categories: 1) one-way vs. round trip - in the former, vehicles may be returned to any legitimate parking spot in the system while in the latter, users are required to return the vehicle to the spot from which it was rented. 2) station-based vs. free-floating - in the former, vehicles can be parked only in system dedicated parking spots whereas in the latter, vehicles can be returned to any on-street parking spot within a predefined polygon. 3) Resource reservations - in some systems, users may be required to reserve in advance a vehicle (vehicle reservation), a parking spot (spot reservation) or both (journey reservation). Reservations may last indefinitely or be limited to specific time windows defined by the system regulations. The main idea behind reservations is to reduce users' uncertainty about the availability of the resources when needed. This, however, may lead in some cases to the unnecessary blocking of highly needed resources. Alternatively, other systems do not require or allow any type of reservations. 4) vehicle type - combustion engine vehicle, electric vehicles, and vehicles based on alternative fueling may imply different needs. For instance, an electric carsharing system is typically station-based, as stations also serve as charging facilities. 5) relocation activities - the actions carried by the system staff to rebalance system inventories may be done mainly at night when the system is essentially idle (static relocations) or throughout the operating day including peak periods (dynamic relocations).

We describe hereafter the type of system at focus in this work, namely a one-way station-based carsharing system implementing complete journey reservations. Under the complete journey reservation regulation, both a vehicle and a destination spot need to be reserved at the time of booking. Notwithstanding, a vehicle can be booked only up to an hour before the rental effectively starts, whereas the destination spot is kept booked until the vehicle is dropped-off by the user. Any reservation can be modified by the customer at any time subject to resource availability. Users pay by the time they have been using the system, starting from the reservation time and until the rental ends. We emphasize that the journey reservation policy should not be viewed as a conventional reservation system. Namely, the exact booking times at which resources are about to be blocked are not known to the operators in advance. Similarly, the exact resource release times are not known in advance, i.e. the times in which vehicles will be taken and returned. From the user's point of view, such rental conditions are particularly favorable as customers are guaranteed a spot at the destination of their choice for an unlimited amount of time whereas the main drawback lies in the inability to book a trip very far in advance. From the operator's point of view, such rules imply that reserved resources cannot be utilized for any other action. In a system with full reservation information (for example in Boyac1 et al. (2017)) where the operator knows the exact time a vehicle will be returned, it could, for instance, utilize this parking spot in between for other vehicles/passengers. Therefore, the operator's main concern is to find the right balance between the attractiveness of the system towards the users and operational costs. A journey reservation policy may potentially enhance the vehicle and spot shortages due to reserved resources, further emphasizing the need to accompany this policy with a reliable and effective relocation policy.

In this paper, we focus on the development of proactive staff-based relocation methods, which aim at both reducing present imbalances in vehicle and spot availability as well as preparing the system for the expected future demand. The contributions of this work are as follows: first, we develop a Markovian model that utilizes reservation information in order to estimate the expected demand losses due to vehicle and spot shortages. Second, we integrate the model in a new proactive and dynamic relocation policy. Third, we compare this new policy to a benchmark no relocation case, to a state-of-the-art inventory rebalancing relocation policy that combines classical approaches in literature and to a centralistic full-knowledge relocation model. Fourth, we report the results of a unique collaboration with the Grenoble carsharing system which allowed us to test various policies in the field. Finally, we further evaluate the performance of the proposed policies using an event-based simulation framework.

The remainder of the paper is organized as follows: Section 2 reviews the carsharing literature and identifies the gaps that this research aims at closing. The proposed dynamic relocation policies and the centralistic full-knowledge relocation model are presented in Section 3. Section 4 introduces the case study based on the Grenoble system and presents the field experiments conducted in collaboration with the system operator. In addition, the results of an extensive simulation experiment are presented and discussed. Concluding remarks and future research directions are given in Section 5.

\section{Literature review}

The literature on carsharing systems can be divided into two main streams. The first stream addresses demandrelated questions through real world carsharing data analysis and the second stream focuses on the supply side, i.e., 
resource planning and management. As this work belongs to the latter, we begin by briefly reviewing data analysis oriented literature and then devote most of this section to literature on the management of carsharing systems.

Real system data has been analyzed using regression models in order to reveal trip patterns and identify the characteristics of successful operation areas for various system types. Data analysis has been conducted for systems implemented in various region of the world, such as: Munich (Schmöller et al. (2015)), Seoul (Kang et al. (2016)), Basel, Zurich (Becker et al. (2017b)) and Montreal (Morency et al. (2008); De Lorimier and El-Geneidy (2013)). Comparing these studies exposes features common to all systems and diverse characteristics that can be explained by different habits, geographical conditions, cultural and economic backgrounds. At a disaggregate level, surveys have been conducted on individuals in order to profile the typical carsharing system member (Becker et al. (2017a,c)). These in-depth analyses allow system operators to target precisely potentially successful operating areas and types of services.

The management of carsharing systems has been addressed at the strategic, tactical and operational levels. For comprehensive reviews on these three planning levels, see Jorge et al. (2013); Laporte et al. (2018). The strategic and tactical decisions levels relate primarily to the location of stations (Correia and Antunes (2012); Boyac1 et al. (2015); Brandstätter et al. (2017)), to fleet sizing (Cepolina and Farina (2014)) and to staff sizing (Kek et al. (2009)).

At the operational level, most of the emphasis has been put on devising methods to redistribute vehicles in the system. Vehicle relocation aims at guaranteeing available vehicles and parking spots where and when needed. For an extensive review regarding relocation mechanisms in one-way carsharing systems, we refer the reader to ?. In the literature, two main approaches have been considered: staff-based relocations, where carsharing system employees move vehicles (Barth et al. (1999); Kek et al. (2006); Boyac1 et al. (2015, 2017); Gambella et al. (2018)), and user-based relocations, where customers adapt their trips according to the operator's suggestions in order to better distribute vehicles in station-based systems (Barth et al. (2004); Di Febbraro et al. (2012, 2019)). These approaches have also been adapted to free-floating systems in ?. Though many studies have focused on staff-based relocations, only a few of them include in their model the routing of the relocators (Nourinejad et al. (2015); Boyac1 et al. (2015, 2017); Gambella et al. (2018)). Active relocation strategies may be also accompanied by passive regulation measures, for instance by controlling vehicle inventories at stations through pricing. This kind of mechanism encourages users to pick-up and drop-off vehicles in preferable stations and has proved effective in reducing the need for staff-based relocations. Various models introducing this feature can be found in Jorge et al. (2015); Waserhole et al. (2013); Waserhole and Jost (2016); Angelopoulos et al. (2018). Other passive regulations such as parking reservation policies (Kaspi et al. (2014, 2016)) have also been considered and have shown to improve system performance. Although staff-based relocation and passive regulations are often implemented together in practice, the effect of combining active and passive measures was rarely studied in the vehicle sharing literature. To the best of our knowledge, the only paper that has considered simultaneously relocations and passive regulations is by ?. Specifically, the paper considers the combination of dynamic relocations and price incentives and demonstrates the ability to tradeoff reward payouts to customers against staff related costs. In this paper, we study for the first time, the combination of dynamic relocations and a complete journey reservation policy with the aim of utilizing reservation information in order to improve staff-based relocation decisions.

Few studies have considered future demand stochasticity to plan for better relocation movements and improve the system's level of service. Nair and Miller-Hooks (2011) formulate a stochastic MIP to make off-line relocation decisions. A joint-chance constraint is introduced to set a lower bound on the future level of service. The performance of the model is tested by dividing the day to several consecutive periods and generating a separate plan for each period. Ma et al. (2018) present a methodology to derive station-specific relocation triggering threshold values in which demand stochasticity is explicitly considered. These thresholds constitute a simple and straightforward way for system operators to detect in real-time overloaded/depleted stations from/to which performing a relocation would be beneficial. However, in their case study, the staff is assumed to be large enough to accomplish all needed vehicle relocations in order to reach the computed threshold values. In addition, staff routing is ignored and the relocation costs are computed per relocation performed, whereas staff related costs are typically a function of the working shifts and not the amount of relocations performed. The authors show that their calculation process generally outputs values different from the ones obtained with classical procedures that ignore stochasticity. Operationally, the introduction of these thresholds results in an overall improved level of service at the cost of minor profit decreases in some stations. More recent studies have put the emphasis on building mid-term relocation plans taking into account stochastic demand. In Wang et al. (2019), a probabilistic approach is introduced to evaluate the risks of a station falling short of resources. For each station, an upper and a lower threshold are defined for each station given fixed alarming probabilities. The relocation plan is then obtained as the solution of an ILP. Besides the level of service improvement due to relocation, the authors 
observe that smaller alarming probabilities lead to an increased number of relocations to be performed even if the ILP optimizes the movements of staff. Song and Murata (2018) rely on stationary distributions of Markov chains to forecast the number of vehicles rented at each station. Based on this, they obtain the surplus and shortages at each station and use a Genetic Algorithm to devise a relocation plan for the next three hours. Their approach is tested in simulation using historical data and improves slightly the state of an already well-working system.

This study focuses on dynamic relocation policies, i.e. relocation operations are executed while the system is active, demand is revealed on-line and the decisions regarding relocations are taken in real-time. Among one of the earliest studies on carsharing, Barth et al. (1999) measure the performance of the carsharing system by the total time users wait for available resources, assuming users are willing to wait unlimitedly. In this work, dynamic decisions are implemented within an event-based simulation in order to assess the impact of relocation decisions on the performance of the system. Three main policies are studied: a policy where relocations are made based on immediate needs and two policies with demand knowledge, one based on historically observed patterns at specific times and the other on average historical demand information. User waiting times are shown to reduce significantly when more information regarding future demand is available. Kek et al. (2006) consider two types of dynamic relocation policies, namely shortest-time and inventory balancing, that are tested within a simulation framework. The former focuses on speed by selecting an origin-destination pair such that an acceptable service state is recovered as soon as possible in at least one of the stations. The second prioritizes vehicle move efficiency and pairs only stations which have complementary needs for vehicles and spots. The inventory-balancing technique is shown to reduce the number of relocations needed while maintaining a comparable level of service while the shortest time relocation policy is shown to decrease zero-vehicle time in stations. The One Vehicle One Spot policy (OVOS), presented in Section 3, combines these two approaches by considering simultaneously stations with resource shortages and the times required for the potential relocation activities. Nourinejad and Roorda (2014) propose a dynamic relocation approach in the form of two complementary optimization models related to vehicle inventory and parking inventory respectively. These models are executed every time a new request appears in the system. Relocations are optimized only with regard to demand already known to the system, namely, users whose booking request was accepted and who have not yet started their trips. Some uncommon features are also introduced: (i) any request acceptance status can be modified from its request time until its rental start time and (ii) station capacity is not a tight operational constraint as system operators may park vehicles outside the station if needed in exchange of extra parking fees. This approach is embedded in a simulation framework to test the impact of trip reservation time on the level of service and fleet size needs. Their case study in Toronto leads to the conclusion that, with their method, an increased trip reservation time can drastically reduce the fleet size needed without reducing the level of service. Weikl and Bogenberger (2015) present a practice-ready hierarchical relocation scheme for free-floating carsharing systems. The scheme includes a mixture of optimization problems and heuristic methods, with a global objective to maximize sales and operator's profit. Static staff-based relocation decisions are made independently for several periods of the day, where the length of each period is a few hours. Real demand realizations during a period are therefore not used to update relocation decisions. Results obtained from a field test led in a Munich carsharing system during three nights spread over three different periods of the year demonstrate the implementability of the proposed scheme and that it outperforms the policy implemented by the operators of the system. However, the significance of the results is limited due to the small number of days tested.

To conclude, with respect to the existing literature and in accordance with gaps identified by Illgen and Höck (2018), this paper addresses three aspects that have not been examined yet. First, the combination of a journey reservation policy and a completely dynamic relocation policy is considered by utilizing real-time reservation information in the relocation decisions. Second, a stochastic representation of the future demand is modeled through a Markovian model that predicts the expected demand loss based on historical data. The model can be fully evaluated off-line and its output can be accessed efficiently during the real-time decision process. Thus, stochastic information is incorporated in real-time decisions in order to generate a proactive relocation policy, where the impact of different decisions can be explicitly quantified and compared. Third, though many of the above mentioned papers validate their approaches through simulation experiments, the result of implementing these approaches in field are rarely reported whereas we have implemented the developed policies in a real-case study environment for a period of three weeks. 


\section{Dynamic relocation policies}

In this section, we present two dynamic staff-based relocation policies designed to generate real-time relocation decisions and an approximated upper bound on the performance of the system. Specifically, in Section 3.1, we present the OVOS policy which integrates simple rules to prioritize stations according to their vehicle and parking availabilities while preferring time efficient relocations. In Section 3.2, we introduce a proactive prediction-based policy that employs a Markovian model to estimate the expected demand loss while taking into account reservation information. Finally, in Section 3.3, we describe a rolling horizon optimization framework for vehicle and personnel relocations. This framework assumes full-knowledge of the daily demand and solutions obtained through it are used to approximate upper bounds on the performance of the system.

\subsection{One Vehicle One Spot Inventory policy (OVOS)}

The aim of this reactive policy is to guarantee at least one available vehicle and one available spot at every station. By reaching this state, the next incoming request is guaranteed to be satisfied. Consequently, the system should respond as fast as possible to any station with no available vehicles or no available parking spots. To this extent, the OVOS policy is a mixture of two well-studied dynamic relocation approaches, namely, inventory balancing and shortest-distance.

In the dynamic setting considered here, a relocation decision is made any time a relocator completes a relocating task, i.e., reaches a relocation destination. In addition, note that when the inventory levels are in a satisfying state, the relocators will not be assigned with new tasks. Therefore, on any occasion that some relocators are idle, a relocation decision is triggered every time the state of the system changes. That is, any time a reservation is made, a vehicle is taken, or a vehicle is returned.

Under the complete journey reservation policy, some vehicles and parking spots may be blocked due to reservations. In other words, the sum of available vehicles and parking spots may be smaller than the capacity of the station. In fact, at some points in time, a station may have no available vehicles and no available parking spots. In addition, in the considered operation mode, relocators are required to reserve a vehicle and a spot, as users do. Therefore, when a relocator is assigned with a task, a vehicle must be available at the origin and a parking spot must be available at the destination.

Let $\beta_{s}$ and $\pi_{s}$ denote the number of available vehicles and available parking spots in station $s$, respectively. At decision points, these values are retrieved and updated by considering on-going relocation tasks. That is, $\beta_{s}$ increases with the number of on-going relocation tasks with destination $s$ and $\pi_{s}$ with the number of on-going relocation tasks that will originate in station $s$, i.e. the number of vehicles that were reserved by relocators but still have not been picked-up. Vehicles reserved by the users are not considered because the exact pick-up times of the users are not known and some users may cancel or modify their reservation.

The decision process is composed of three steps: classification of potential origins and potential destinations, prioritization of potential origin-destination pairs and selection of an origin-destination pair according to a shortest distance criterion. At decision points, stations are first classified based on their potential to be an origin or a destination of a relocation. In Table 1, four classes of origins and destinations are presented, denoted by $O_{0}, O_{1}, O_{2}, O_{3}$ and $D_{0}$, $D_{1}, D_{2}, D_{3}$ respectively. These classes define priorities regarding the stations vehicles should be relocated to and from according to their current available resources inventory states. Note that a station having $\beta_{s} \leq 1$ is not considered as a potential relocation origin, as it has either the minimum required level of available vehicles or no available vehicles. Similarly, a station having $\pi_{s} \leq 1$ cannot be considered as a potential relocation destination. Next, sets of origin destination pairs are generated and prioritized according to the priority levels displayed in Table 2. Note that priorities are presented in decreasing order for selecting an origin-destination pair, i.e. priority 1 level contains the most urgent pairs of stations for a relocation. Lastly, an origin-destination pair is selected from the highest non-empty priority level. Specifically, among all candidate origin-destination pairs having the same (highest) priority, we select the pair that minimizes relocation duration. This duration is the sum of the time required for the relocator to reach the origin station and the driving time from the origin to the destination. 


\begin{tabular}{|l|l|}
\hline Relocation origin classes & Relocation destination classes \\
\hline$O_{0}=\left\{s \in S \mid \beta_{s} \geq 2, \pi_{s}=0\right\}$ & $D_{0}=\left\{s \in S \mid \beta_{s}=0, \pi_{s} \geq 2\right\}$ \\
\hline$O_{1}=\left\{s \in S \mid \beta_{s} \geq 3, \pi_{s}=1\right\}$ & $D_{1}=\left\{s \in S \mid \beta_{s}=1, \pi_{s} \geq 3\right\}$ \\
\hline$O_{2}=\left\{s \in S \mid \beta_{s} \geq 3, \pi_{s} \geq 2\right\}$ & $D_{2}=\left\{s \in S \mid \beta_{s} \geq 2, \pi_{s} \geq 3\right\}$ \\
\hline$O_{3}=\left\{s \in S \mid \beta_{s}=2, \pi_{s} \geq 1\right\}$ & $D_{3}=\left\{s \in S \mid \beta_{s} \geq 1, \pi_{s}=2\right\}$ \\
\hline
\end{tabular}

Table 1: OVOS policy - origin and destination classes

\begin{tabular}{|l|l|}
\hline Priority & OD pair sets \\
\hline 1 & $\left\{(o, d) \mid o \in O_{0}, d \in D_{0}\right\}$ \\
\hline 2 & $\left\{(o, d) \mid o \in O_{0}, d \in D_{1}\right\},\left\{(o, d) \mid o \in O_{1}, d \in D_{0}\right\}$ \\
\hline 3 & $\left\{(o, d) \mid o \in O_{0}, d \in D_{2} \cup D_{3}\right\},\left\{(o, d) \mid o \in O_{2} \cup O_{3}, d \in D_{0}\right\}$ \\
\hline 4 & $\left\{(o, d) \mid o \in O_{1}, d \in D_{1} \cup D_{2}\right\},\left\{(o, d) \mid o \in O_{2}, d \in D_{1}\right\}$ \\
\hline
\end{tabular}

Table 2: OVOS policy - relocation pair prioritization

As can be observed in Table 2, the highest priority is given to pairs that contain an origin with no available parking spot and a destination with no available vehicle. Next in priority are pairs in which one station has no available vehicle (resp. parking spot) and the other station has only one available parking spot (resp. vehicle). Following are pairs in which one of the stations has no available vehicle/parking spot and the last priority level considers pairs in which at least one of stations has only a single vehicle/parking spot available. We note that the prioritization is motivated by the lack of resources and not by the available resources. For example, a station having one available vehicle and two available parking spots will have the same priority as a station with one available vehicle and four available spots. One could argue that the latter should be preferred, as selecting such station as a destination would lead to more equitable distribution of available parking spots. However, this kind of consideration may lead to longer relocation times and therefore a less efficient allocation of the relocators' work time.

To conclude, the significance of the OVOS policy is in combining two important factors related to relocation: inventory management and relocation duration, while its simple structure makes it easy to implement and communicate to the operators. In Section 4.3, we will demonstrate the effectiveness of this policy as compared to the operator's policy. We note that in systems where stations exhibit highly asymmetric demand rates, target levels higher than one might need to be considered. However, as in most carsharing systems station capacities are rather low, a target level of one is often the most suitable.

\subsection{Markovian estimation policy}

In this strategy, the impact of relocation choices on the future level of service of the system is quantified in order to make proactive relocation decisions. For this purpose, we formulate a Markovian model that utilizes historical demand information in order to estimate near-future shortage of vehicles and parking spots at a station. Such a modeling approach was initially proposed by ? to measure user dissatisfaction in bike-sharing systems and was later adopted by several studies in the vehicle sharing literature. However, none of the previous studies accounted for reservation information in the model. Such information is important especially for short term estimation periods as it reduces uncertainty regarding vehicles and parking spots that are about to become available. In what follows, we describe a Markovian model for a single station that incorporates reservation information, which complexifies the chain structure, and we explain how the output of the model is used for real-time relocation decisions. The Markovian model is applied for all stations in the system and for any potential decision period. To simplify the presentation of the model, we consider next a given station $s$ at a given decision time $t$. This allows us to temporarily omit indices representing the stations and the decision time. Specifically, without loss of generality, the decision time occurs at the beginning of the prediction horizon.

Consider a single station with a given number of parking spots, denoted by $C$. Under the complete journey policy, each parking spot may be in one of the five following states: available vehicle (av); reserved vehicle for a one-way trip (rv); reserved vehicle for a round trip (rv'); reserved parking spot (rp); available parking spot (ap). 
The distinction between (rv) and (rv') is necessary to represent accurately station inventory dynamics. Indeed, the pick-up of a vehicle reserved for a round-trip does not free up a spot, contrary to the pick-up of a vehicle reserved for a one-way trip. We denote therefore the state of station $s$ at time $t$ by the quadruplet $\left(x_{a v}, x_{r v}, x_{r v^{\prime}}, x_{r p}\right)$ where $x_{a v}$ represents the number of available vehicles, $x_{r v}$ and $x_{r v^{\prime}}$ represent the number of reserved vehicles for one-way trips and round-trips respectively, and $x_{r p}$ represents the number of reserved spots. As capacity is fixed, the number of available parking spots is directly given by $C-x_{a v}-x_{r v}-x_{r v^{\prime}}-x_{r p}$.

The transitions between the states of the station are due to seven events that may occur at the station: (i) spot booking when a one-way trip request to the station is accepted; (ii) vehicle booking when a one-way trip request from the station is accepted; (iii) vehicle booking when a round trip request at the station is accepted; (iv) vehicle pick-up when a one-way rental starts; (v) vehicle pick-up when a round trip rental starts (vi) vehicle drop-off when a one-way rental ends; (vii) vehicle drop-off when a round trip rental ends.

We model the evolution of the station as a continuous time Markov chain. Particularly, we assume that the arrival of vehicle requests at a station follows time heterogeneous Poisson processes with rates $\lambda_{v}(t)$ for one-way trips and $\lambda_{v}^{\prime}(t)$ for round trips. Similarly, the arrival of parking spot requests at a station also follows a time heterogeneous Poisson process with rate $\lambda_{p}(t)$. This rate corresponds only to the parking spot request rate of one-way trips. In the case of round trips, the destination spot is in fact also the starting spot and is therefore directly booked when the corresponding vehicle is reserved. Further on, we assume that the time between the users' reservation and their arrival at the station is exponentially distributed with mean $\mu_{v}(t)^{-1}$ for one-way trip requests and that the spot reservation duration at the station is exponentially distributed with mean $\mu_{p}(t)^{-1}$ and $\mu_{p}^{\prime}(t)^{-1}$ for one-way trips and round trips respectively. The transition rates out of state $\left(x_{a v}, x_{r v}, x_{r v^{\prime}}, x_{r p}\right)$ are summarized in Table 3. We highlight that this Markov chain structure does not explicitely represent the pick-up of a vehicle in the case of round trip request. Such event does not directly impact the state of the station as the corresponding spot remains blocked to other requests until the vehicle is returned. Therefore, we ignore this event and rather directly model the transition (of rate $\mu_{p}^{\prime}(t)$ ) between the reservation of a vehicle for a round trip request and its drop-off at the end of the corresponding rental.

\begin{tabular}{|l|l|l|l|}
\hline Event & Current state & Next state & Trans. rate \\
\hline $\begin{array}{l}\text { Booking } \\
\text { of available vehicle } \\
\text { for a one-way trip }\end{array}$ & $\begin{array}{l}\left(x_{a v}, x_{r v}, x_{r v^{\prime}}, x_{r p}\right) \\
x_{a v}>0\end{array}$ & $\left(x_{a v}-1, x_{r v}+1, x_{r v^{\prime}}, x_{r p}\right)$ & $\lambda_{v}(t)$ \\
\hline $\begin{array}{l}\text { Booking } \\
\text { of available vehicle } \\
\text { for a round trip }\end{array}$ & $\begin{array}{l}\left(x_{a v}, x_{r v}, x_{r v^{\prime}}, x_{r p}\right) \\
x_{a v}>0\end{array}$ & $\left(x_{a v}-1, x_{r v}, x_{r v^{\prime}}+1, x_{r p}\right)$ & $\lambda_{v}^{\prime}(t)$ \\
\hline $\begin{array}{l}\text { Pick-up } \\
\text { of booked vehicle } \\
\text { for a one-way trip }\end{array}$ & $\left(x_{a v}, x_{r v}, x_{r v^{\prime}}, x_{r p}\right)$ & $\left(x_{a v}, x_{r v}-1, x_{r v^{\prime}}, x_{r p}\right)$ & $x_{r v} \mu_{v}(t)$ \\
\hline $\begin{array}{l}\text { Drop-off of vehicle } \\
\text { after a one-way trip }\end{array}$ & $\begin{array}{l}\left(x_{a v}, x_{r v}, x_{r v^{\prime}}, x_{r p}\right) \\
x_{r p}>0\end{array}$ & $\left(x_{a v}+1, x_{r v}, x_{r v^{\prime}}, x_{r p}-1\right)$ & $x_{r p} \mu_{p}(t)$ \\
\hline $\begin{array}{l}\text { Drop-off of vehicle } \\
\text { after a round trip }\end{array}$ & $\begin{array}{l}\left(x_{a v}, x_{r v}, x_{r v^{\prime}}, x_{r p}\right) \\
x_{r v^{\prime}}>0\end{array}$ & $\left(x_{a v}+1, x_{r v}, x_{r v^{\prime}}-1, x_{r p}\right)$ & $x_{r v^{\prime}} \mu_{p}^{\prime}(t)$ \\
\hline $\begin{array}{l}\text { Booking } \\
\text { of available spot } \\
\text { for a one-way trip }\end{array}$ & $\begin{array}{l}\left(x_{a v}, x_{r v}, x_{r v^{\prime}}, x_{r p}\right) \\
x_{a v}+x_{r v}+x_{r v^{\prime}}+x_{r p}<C\end{array}$ & $\left(x_{a v}, x_{r v}, x_{r v^{\prime}}, x_{r p}+1\right)$ & $\lambda_{p}(t)$ \\
\hline- & $\left(x_{a v}, x_{r v}, x_{r v^{\prime}}, x_{r p}\right)$ & & 0 \\
\hline
\end{tabular}

Table 3: Transitions between states in the independent station Markov chain model

As an example, the resulting continuous time Markov chain for a station with two parking spots is illustrated in Figure 1. Consider state $(1,0,0,0)$, the state in which the station has 1 available vehicle, 0 reserved vehicles (for round-trip and one way-trip), 0 reserved spots and 1 available spot. The potential transitions from this state are the following: the available vehicle is booked for a one-way trip $\left(\lambda_{v}\right.$, to $\left.(0,1,0,0)\right)$; the available vehicle is booked for a round trip $\left(\lambda_{v}^{\prime}\right.$, to $\left.(0,0,1,0)\right)$; the available spot is booked for a one-way trip $\left(\mu_{p}\right.$, to $\left.(1,0,0,1)\right)$. The potential transition to this state are as follows: a vehicle in a one-way trip is dropped-off at the station and the reserved spot is filled $\left(\mu_{p}\right.$, from 
$(0,0,0,1))$, a vehicle is picked-up for a one-way trip and frees a spot $\left(\mu_{v}\right.$, from $\left.(1,1,0,0)\right)$, a vehicle performing a round trip is returned to its reserved spot and the vehicle becomes available $\left(\mu_{p}^{\prime}\right.$, from $\left.(0,0,1,0)\right)$.

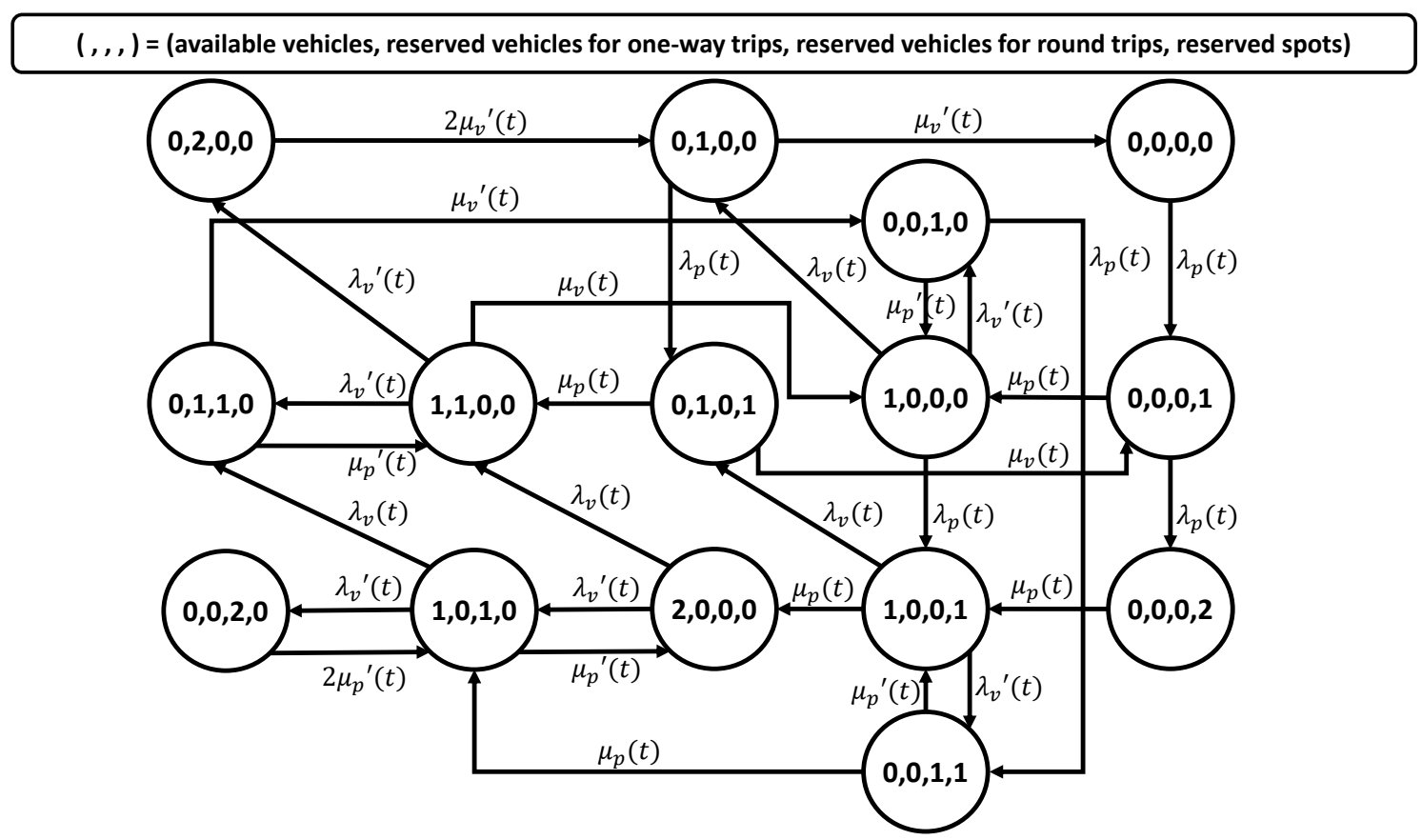

Figure 1: Continuous time Markov chain for a station with a capacity of two parking spots

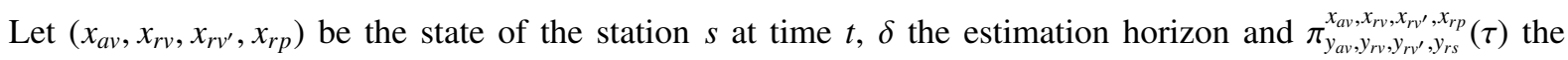
probability that the station is in state $\left(y_{a v}, y_{r v}, y_{r v^{\prime}}, x_{r s}\right)$ at time $\tau, t \geq \tau \geq t+\delta$. The expected demand loss due to vehicle and parking shortage at station $s$ during $\delta$ is:

$$
\begin{aligned}
E L\left(x_{a v}, x_{r v}, x_{r v^{\prime}}, x_{r p}\right)=\int_{t}^{t+\delta} \sum_{i=0}^{C} \sum_{j=0}^{C-i} \sum_{k=0}^{C-i-j} \pi_{0, i, j, k}^{x_{a v}, x_{r v}^{t}, x_{r v^{\prime}}, x_{r p}}(\tau)\left(\lambda_{v}+\lambda_{v}^{\prime}\right)(\tau) d \tau & \\
& +\int_{t}^{t+\delta} \sum_{i=0}^{C} \sum_{j=0}^{C-i} \sum_{k=0}^{C-i-j} \pi_{i, j, k, C-i-j-k}^{x_{a v}, x_{r v}, x_{r v^{\prime}}, x_{r p}}(\tau)\left(\lambda_{p}(\tau)\right) d \tau
\end{aligned}
$$

In the first term of the sum, the term in the integral represents the rate of requests that cannot be fulfilled due to shortages in available vehicles, obtained by multiplying the probability for vehicle shortage at time $\tau$ by the arrival rate of requests for vehicles $\lambda_{v}(\tau)+\lambda_{v}^{\prime}(\tau)$. Similarly, in the second term of the sum, the term in the integral represents the rate of requests that cannot be fulfilled due to shortage in available parking spots, obtained by multiplying the probability for parking spot shortage at time $\tau$ by the arrival rate of requests for parking spots $\lambda_{s}(\tau)$. The evaluation of the expected demand loss is numerically obtained by applying the approximation method proposed in ?. We note that in a single run of the procedure, the expected demand losses for all possible initial states are obtained. For real-time implementation, we precalculate the expected demand loss for every station, every period of the day and every possible initial state. Specifically, we discretize the day into time periods of length $\Delta$ and given a prediction horizon $\delta$, we run the approximation method for every station and every period.

In the considered operation mode, relocators are required to reserve a vehicle and a spot. Therefore, a station is considered as a potential origin for a relocation only if at the decision time $x_{a v} \geq 0$. If the origin station is in state $\left(x_{a v}, x_{r v}, x_{r v^{\prime}}, x_{r p}\right)$ when the relocation task is assigned, it will transition to state $\left(x_{a v}-1, x_{r v}+1, x_{r v^{\prime}}, x_{r p}\right)$. That is, an available vehicle has been reserved, thus decreasing the number of available vehicles by one and increasing the number 
of reserved vehicles by one. $O\left(x_{a v}, x_{r v}, x_{r v^{\prime}}, x_{r p}\right)$ denotes the expected avoided demand loss during the estimation period if the station is selected to be an origin of a relocation and is defined as follows:

$$
O\left(x_{a v}, x_{r v}, x_{r v^{\prime}}, x_{r p}\right)=E L\left(x_{a v}, x_{r v}, x_{r v^{\prime}}, x_{r p}\right)-E L\left(x_{a v}-1, x_{r v}+1, x_{r v^{\prime}}, x_{r p}\right)
$$

Equivalently, a station is considered as a potential destination for a relocation only if at the decision time $x_{a v}+x_{r v}+$ $x_{r v^{\prime}}+x_{r p}<C$. Similarly, if the destination station is in state $\left(x_{a v}, x_{r v}, x_{r v^{\prime}}, x_{r p}\right)$ when the relocation task is assigned, the station will transition to state $\left(x_{a v}, x_{r v}, x_{r v^{\prime}}, x_{r p}+1\right)$. Then, $D\left(x_{a v}, x_{r v}, x_{r v^{\prime}}, x_{r p}\right)$, the expected avoided demand loss during the estimation period if the station is selected to be the destination of a relocation, is given by:

$$
D\left(x_{a v}, x_{r v}, x_{r v^{\prime}}, x_{r p}\right)=E L\left(x_{a v}, x_{r v}, x_{r v^{\prime}}, x_{r p}\right)-E L\left(x_{a v}, x_{r v}, x_{r v^{\prime}}, x_{r p}+1\right)
$$

At a decision time, the expected avoided losses are calculated for all stations, only with respect to their current states. For the sake of clarity, we omit hereafter the state arguments and introduce a station index to differentiate between stations. That is, the expected avoided loss at station $s$ if it is selected as an origin or as a destination for a relocation, are denoted by $O^{s}$ and $D^{s}$, respectively. The expected avoided demand loss due to a relocation from station $s_{1}$ to station $s_{2}$ is the sum $O^{s_{1}}+D^{s_{2}}$. Under this policy, any pair of stations $\left(s_{1}, s_{2}\right)$ for which $O^{s_{1}}+D^{s_{2}}>0$, is considered as a candidate for a relocation. In cases where no candidates exist, no relocation is performed.

Finally, in selecting a pair from the set of candidates, we wish to balance the expected avoided demand loss $O / D$ against the time required to execute the relocation task. In order to do so, we calculate the expected avoided demand loss per time unit spent relocating. Specifically, let move $\left(s_{1}\right)$ the time required for the relocator to reach station $s_{1}$ from its current location at decision time $t$ and $\operatorname{drive}\left(s_{1}, s_{2}\right)$ the time required for the relocator to drive a vehicle from $s_{1}$ to $s_{2}$. The total time required to relocate a vehicle from $s_{1}$ to $s_{2}$ is given by move $\left(s_{1}\right)+$ drive $\left(s_{1}, s_{2}\right)$ and the expected avoided demand loss per time unit spent relocating is given by $\left(O^{s_{1}}+D^{s_{2}}\right) /\left(\operatorname{move}\left(s_{1}\right)+\right.$ drive $\left.\left(s_{1}, s_{2}\right)\right)$. The relocation pair that has the highest expected avoided demand loss per time unit spent relocating is selected as the next relocation to perform. Let $\mathrm{P}$ be the set of candidate pairs for a relocation, we select the relocation pair $\left(o^{\star}, d^{\star}\right)$ such that:

$$
\left(o^{\star}, d^{\star}\right)=\arg \max _{\left(s_{1}, s_{2}\right) \in P} \frac{O^{s_{1}}+D^{s_{2}}}{\operatorname{move}\left(s_{1}\right)+\operatorname{drive}\left(s_{1}, s_{2}\right)}
$$

As in the OVOS policy, a relocation decision is made any time a relocator completes a relocating task or when the state of the system is changed while some relocators are idle.

In order to make the on-line relocation pair decisions in real time, the Markovian Estimation policy requires an off-line stage. In this pre-processing stage, we calculate the expected demand loss over the horizon $\delta$ for any time of the day and any possible initial state for each station, following the calculation method from ?. The day is divided in periods of 5 minutes starting respectively at $\{0,5,10, \ldots, 1430,1435\}$. Given the historical rate values and for every station $s$, every initial state $\left(x_{a v}, x_{r v}, x_{r v^{\prime}}, x_{r s}\right)$ and every period start time $t_{s t a r t}$, we calculate $E L_{t_{s a r t}}^{s}\left(x_{a v}, x_{r v}, x_{r v^{\prime}}, x_{r s}\right)$ and store its value. We explain hereafter how these stored values are used in real-time later. At any decision point $t$ (i.e. with an idle relocator), the current inventory state of all stations is first retrieved. Second, for all candidate stations to be an origin/destination for a relocation, $O / D$ is calculated by retrieving $E L$ for the relevant station states at the period containing $t$. Third, the idle relocator is assigned with the best-origin destination pair, i.e. the one with the highest expected avoided demand loss per time unit spent relocating. The on-line process is represented in Figure 2. 


\section{ON-LINE ALGORITHM}

Retrieve the current inventory of the station from the monitoring of the system

Is the station a candidate origin for relocation, i.e. has an available vehicle?

Calculate the avoided demand loss if a relocation was to start from the candidate station

Is the station a candidate destination for relocation, i.e. has an available veh.?

Calculate the avoided demand loss if a relocation was to end at the investigated station

Select the pair with the highest avoided demand loss per time spent relocating

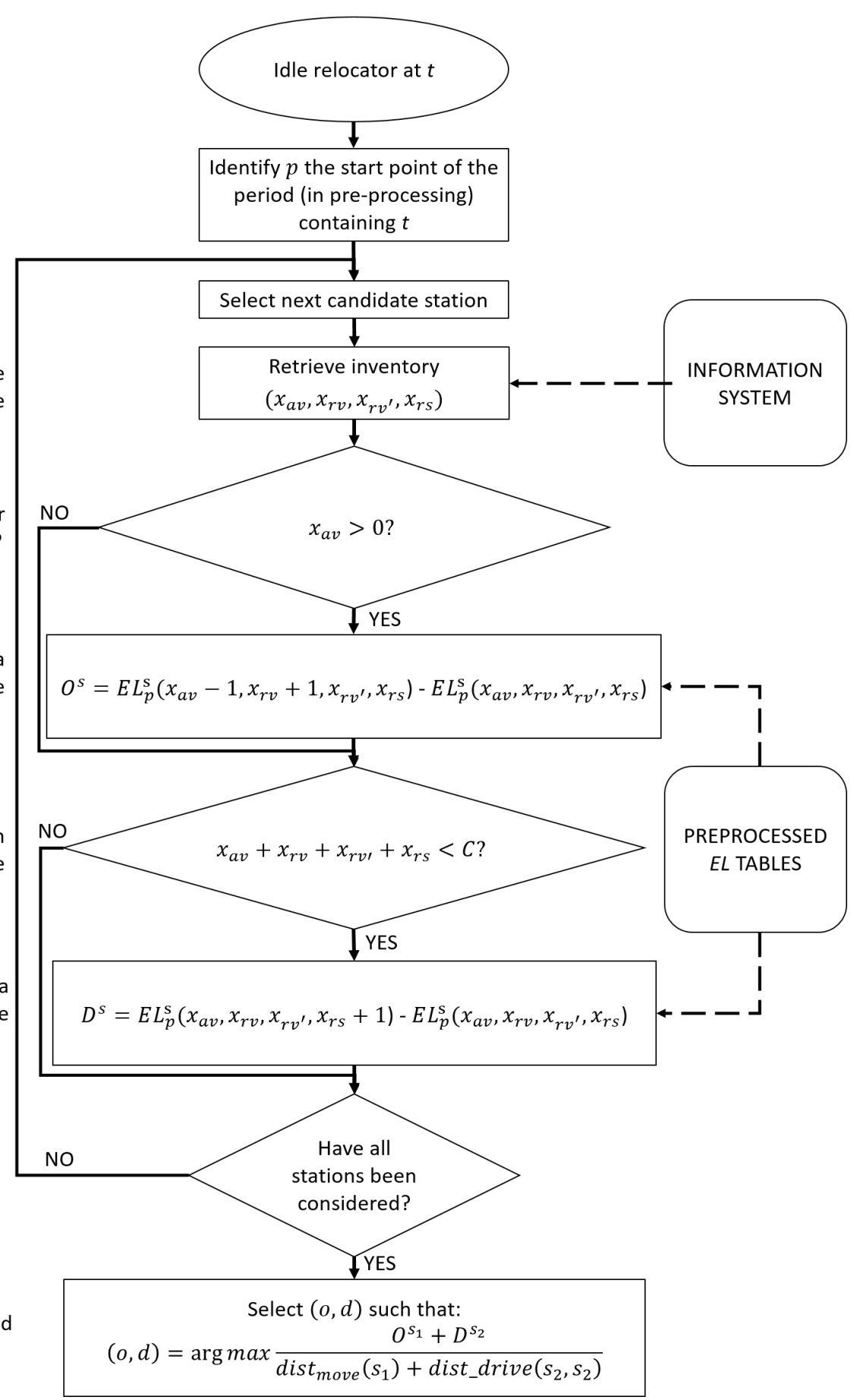

Figure 2: Markovian Estimation policy on-line decision algorithm flowchart 


\subsection{An approximate upper bound for benchmarking policy performance}

As will be demonstrated in Section 4, the dynamic staff-based relocation policies presented above have shown to perform well in terms of the number of accepted requests. In order to further evaluate the quality of these policies, we wish to bound the potential additional improvement that may be obtained by any other dynamic relocation policy.

For this purpose, we employ a centralistic approach with in-advance full knowledge of the demand. Recall that in a dynamic setting, decisions are taken without knowing the exact future demand realizations. Furthermore, under the considered operational regulations, any user request that can be served is accepted. However, from a global perspective, the system might be better off not accepting certain requests. Clearly, a centralistic approach that simultaneously decides which requests to accept and which relocations to perform, having full knowledge of future demand, will perform better than any dynamic policy.

To that end, we developed a mathematical model that we present in details in Appendix A. Specifically, we adapt the operations optimization model presented in Boyac1 et al. (2017) to the specific settings of carsharing systems implementing complete journey reservation. The model is a time-indexed MILP formulation designed to maximize the number of accepted requests. It receives as an input the full daily demand data, including: time of request, starting and ending times of the requested rentals and their starting and ending locations. The primary objective of the model is to maximize the number of accepted request, subject to flow conservation constraints of vehicles and relocators, capacity constraints, parking and vehicle reservations constraints. The model is solved via a rolling horizon framework, that is, the demand input consists of several consecutive days and the model is solved iteratively, day-by-day. Specifically, the end locations of the vehicles in a given day are taken as the initial locations of the vehicles in the following day.

While the time data related to the demand and the movement of vehicles and relocators is given in fine resolution, a rougher time discretization is applied in the optimization model for tractability reasons. Specifically, the durations of the rentals and the relocation durations were rounded up to the closest discrete value. As a result, the output of the model is not theoretically an upper bound on the number of accepted requests, but instead only a good approximation of it.

\section{Experimental Results}

The dynamic relocation policies described in the previous section were tested taking the Grenoble carsharing system as a case study. In Section 4.1, we describe the characteristics of the Grenoble system and the data derived from it for the purpose of our experiments. In section 4.2, we describe the setup and results of fields experiments we conducted in collaboration with the operators of the system. Section 4.3 presents an extensive numerical experiment conducted in order to further validate the results obtained in the field.

\subsection{Case Study}

The Grenoble carsharing system was inaugurated in September 2014 and was operational until November 2017. It implemented the complete journey reservation policy. Note that similar reservation based policies are currently applied in other cities like Toyota City and Bangkok. The system consisted of 27 stations dispersed in the city center and some surrounding suburbs, including the university campus. In total, 121 parking spots were installed in the system. The capacities of the stations ranged from three to eight parking spots, where the majority of the stations had a capacity of four. Figure 3 displays the locations of the stations and their capacities over the Grenoble map.

The vehicle fleet consisted of 70 ultra-compact electric vehicles, although in practice, only 40 to 55 vehicles where available for rent at a given time due to maintenance requirements. Two types of vehicles were deployed in the system, the Toyota i-ROAD and the Toyota COMS. The first is a two-seater, three-wheel vehicle with rear wheel steering that required basic training and the second is a one-seater four-wheel vehicle with a small trunk. The vehicles' maximum speed corresponds to the speed limit in urban areas in France, $50 \mathrm{~km} / \mathrm{h}$, thus forbidding them to enter the Grenoble ring road highway. Both types of vehicles are electric and their common full battery range is about 50 kilometers. Therefore, rentals are considered terminated only when vehicles are plugged to the station docks. The system operated 24 hours a day, 7 days a week. Usage fee was fixed to $1 €$ per 15 minutes, independently of the distance travelled.

Also, in reality, vehicles are required to have a minimum level of battery to be available for rent. In the simulation part of this case study, we disregarded battery restrictions. Preliminary simulation runs have shown that in the case study, battery limitations only marginally impact the results. Illgen and Höck (2018) supports this fact by demonstrating 


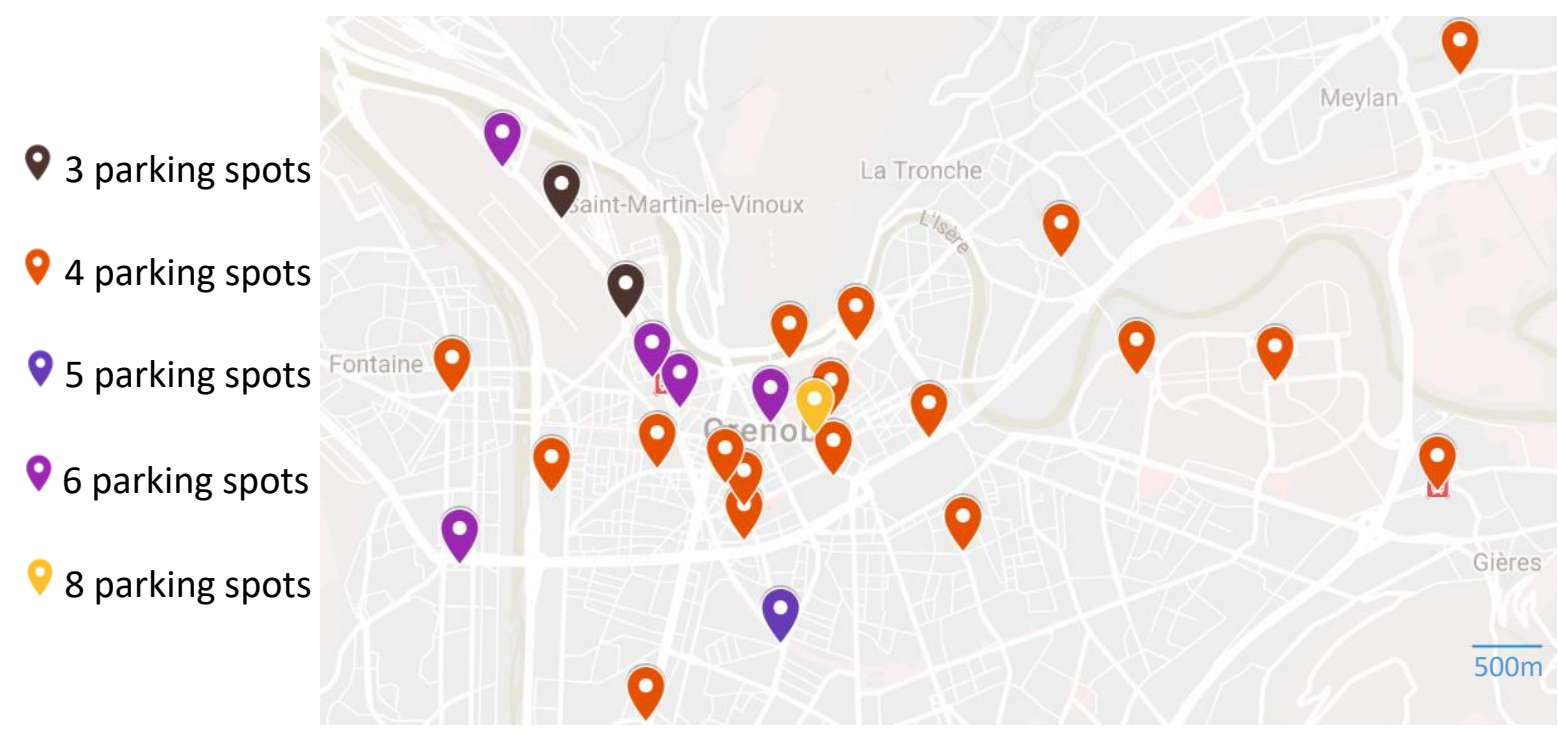

Figure 3: Grenoble case study station network

that EV carsharing may perform as good as classic carsharing with some particular demand characteristics as well as fleet and vehicle settings such as ours. If the battery charging and range requirements are not negligible, EV-based systems should have more cars with shorter ranges (and therefore less expensive) with a well-developed charging infrastructure. In such cases, the limitations coming from EVs are well mitigated. Also, a proper analysis regarding energy consumption and trip distances as in Brendel et al. (2018) facilitates the smart deployment of EVs for the operator in carsharing systems and appeases the users' potential battery anxiety.

Prior to our study, relocation was performed by two staff members on weekdays between 9 a.m. and 5 p.m., i.e. after the start of the demand peak and before its end. Relocation decisions were taken by the relocators with the help of a decision-support tool, a web API identifying critical stations based on the current state of the system. The relocators' decisions were often motivated by other side requirements as they were also responsible for vehicle maintenance and for the training of new users. Also, no end-of-the-day relocations (to reset the system to a good state) were performed during the low-usage period of the system, namely at night. Such relocation strategies are out of the scope of this study. Besides, introducing relocation night shifts comes at the price of increased salaries due to arduous working conditions. Consequently, in our study, the early morning fleet positioning for the next day is influenced by the previous days' operations and relocations. The effect of resetting vehicle distribution at night vs. dynamic relocation policies was previously examined by the authors in Repoux et al. (2015).

The operators of the system have shared with us the trip transaction data ranging from September 2014 to April 2017. We have preprocessed data and removed unrealistic transactions or transactions that were irrelevant to our analysis. Specifically, we ignored relocator transactions and transactions trips with very short or very long rental durations. In addition, round trips with durations shorter than 5 minutes were also ignored.

Figure 4 presents the distribution of the demand over the periods of the day. The daily distribution shows that the majority of the demands were evenly spread between 7 a.m. to 8 p.m., with some slight decreases in the late mornings and the early afternoons. Figure 5 and 6 display the spatial distributions of demands over the network. Naturally, stations in the heart of Grenoble (stations 1, 2, 3, 4, 5 and 7) exhibited the highest rental intensity while suburban locations (stations 22, 23, 24 and 26) were least used. 


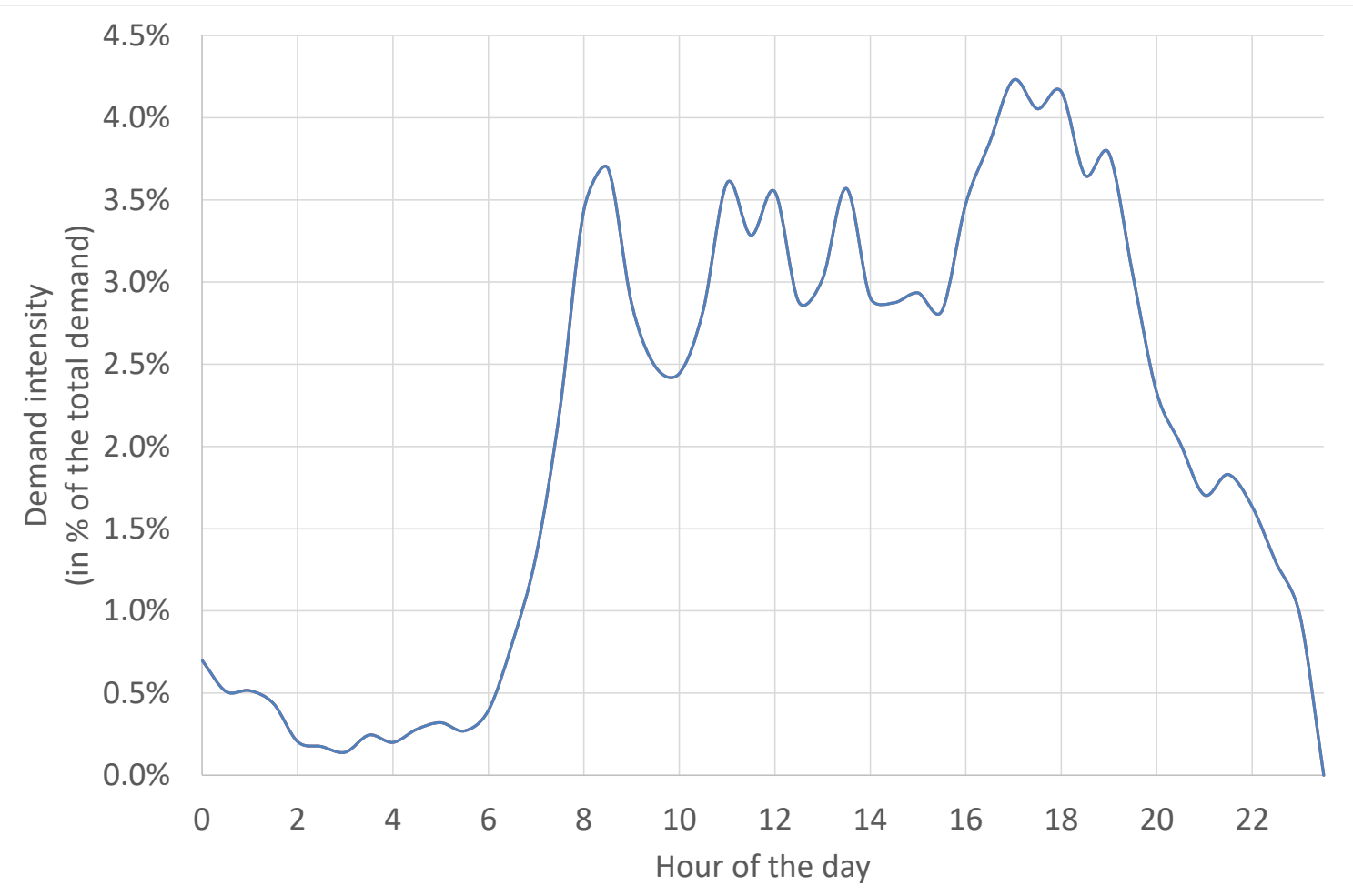

Figure 4: Hourly observed demand variations during the day in the Grenoble system

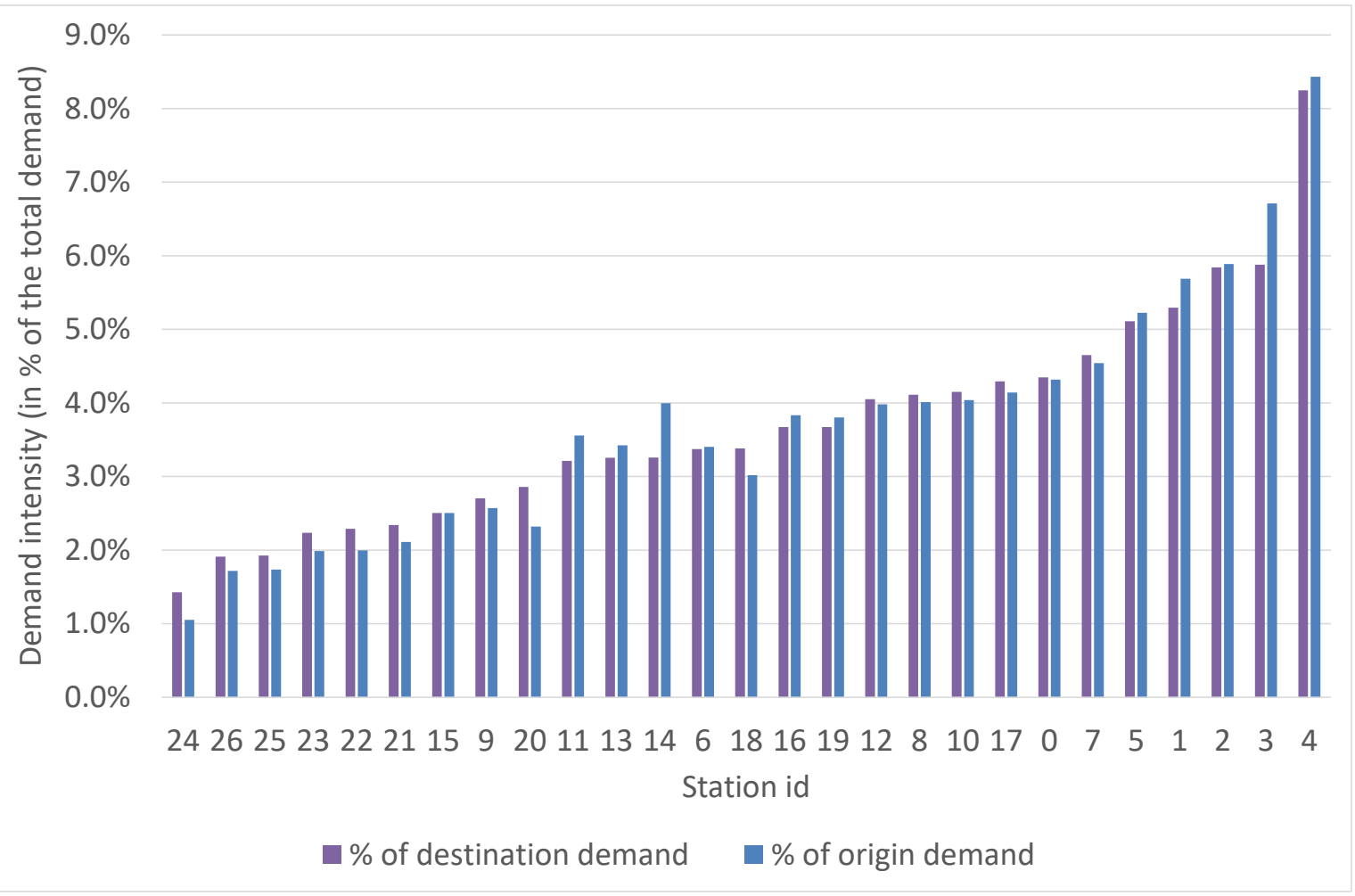

Figure 5: Distribution of observed demand by station 


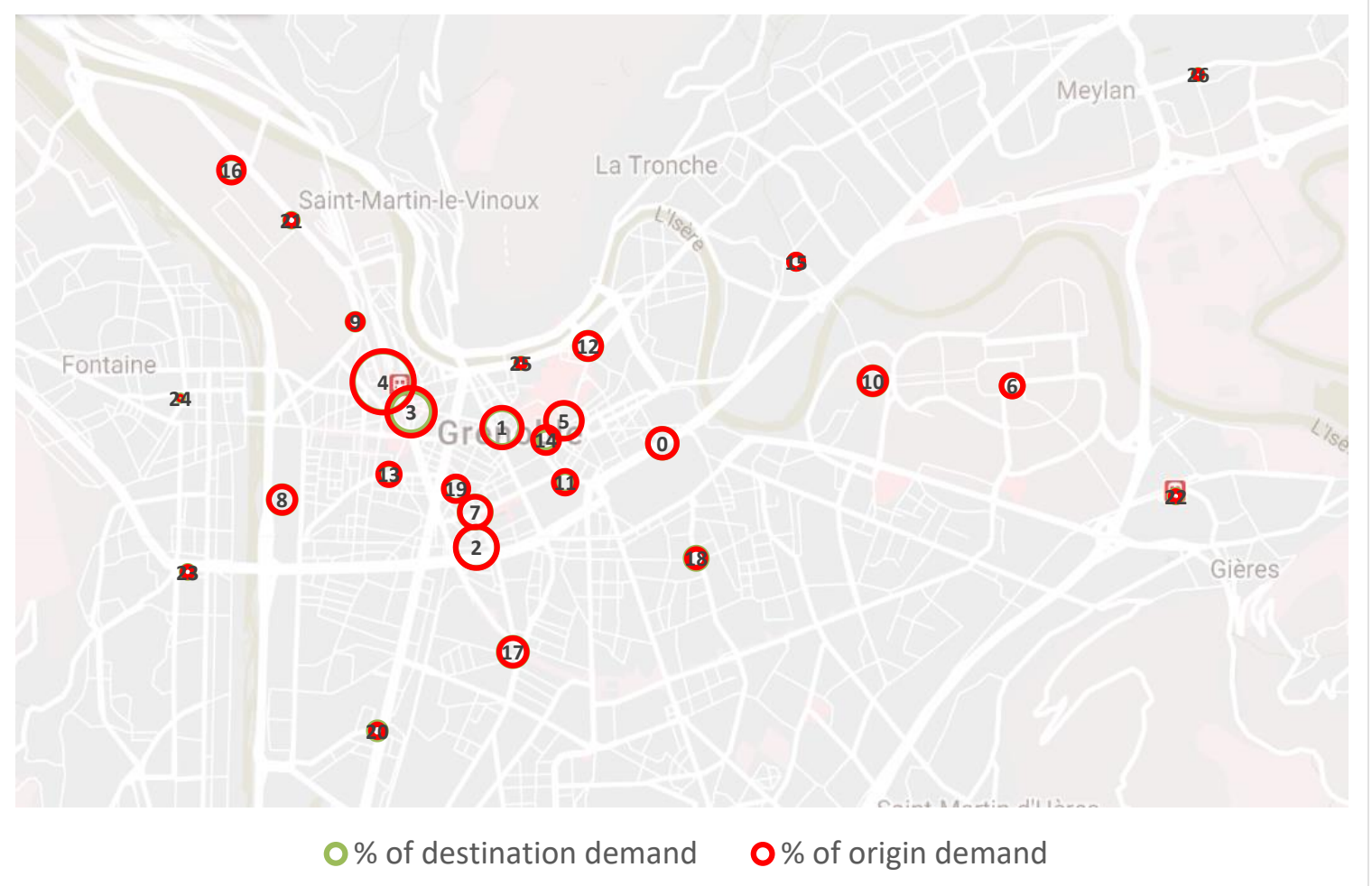

Figure 6: Distribution of observed demand by station. The diameter of the bubbles is proportional to demand intensity.

Next, we used the processed trip transaction data to estimate hourly renting and returning rates at each station. This time resolution was chosen so as to describe precisely enough dynamic variations while having a sufficient number of data leading to statistically meaningful values. First, for each station and each time period, we counted the total number of rents/returns and divided by the number of transactions in the data. To smoothen the observed rates, the rate of each time period is calculated as a moving average considering the preceding and following periods.

Two additional parameters important for our experiments are the vehicle and parking spot reservation durations. They represent the time elapsed from customer booking to vehicle pick-up and to vehicle drop-off, respectively. The vehicle reservation duration is most likely user-specific, however, user IDs were not provided in the trip transaction data. We assume that vehicle reservation durations are neither station-specific nor time-specific. That means we suppose that all users have the same behavior regarding their access to vehicle time independently of their location or their reservation time. Spot reservation duration may depend on the considered origin-destination pair and reservation time. Due to the limited number of transactions for some ODs, we converged to destination-specific reservation durations. Doing so, we suppose that the spot reservation duration is approximately the same at any time of the day and depends only on the location of the destination station.

Statistical tests show that the spot and vehicle reservation events may follow a Poisson process for $66 \%$ of the 1-hour periods in a day. However, the inter-event durations for the other events do not follow exponential laws. Therefore, we approximate the real distributions to exponential ones for the needs of the Markovian model. The effectiveness of the method is later demonstrated in Section 4.3.

\subsection{Field implementation and testing}

The collaboration with the Grenoble carsharing system provided us a unique opportunity to test our proposed policies in the field. Two field experiments were set in June and September-October 2017. The objectives of these experiments were as follows: 1) Understand how the system operates and what issues are met in real operations; 2) Test the performance of the system under higher demand loads; 3) Test and document the effect of various relocation strategies; 4) Collect data that is unavailable in the information system, in particular, the fraction of unfulfilled requests and the relocators' traveling times. 
These field tests were also an opportunity for the system operator to test a wide variety of other regulations. Consequently, the policies presented in this paper were not the only ones tested. We briefly comment on other policies tested in our discussion of the results.

The preparation for the field test included the introduction of mechanisms to increase demand significantly, the adjustment of relocators' schedules and the design of a web interface for relocation tasks assignment. We elaborate about these procedures next. We have set as a target to reach 100 demands per day during the field experiment, that is, to increase the average daily demand observed in the period by about $200 \%$. This target was selected as it was more or less the demand level that the system operators were aiming at reaching. In addition, closing the gap between the actual demand level and the targeted 100 demands, via extra exogenous demand, seemed realistic with the resources that were at our disposal during the experiment. We have increased demand through two mechanisms: first, some frequent users of the system were offered free usage during the period of the field experiment in order to increase demand by regular users. Second, we hired temporary employees to artificially generate more rentals during the day. In the rest of this section, we refer to these employees as drivers to differentiate them from regular users. The rentals performed by the drivers were generated in compliance with the demand patterns exhibited in the trip transaction data. In addition, extra round trips were virtually performed by blocking some vehicles at their spots for a period of time through the control management system. We emphasize that no specific information about the artificially added demand was available to the tested relocation algorithms. Besides increasing the demand to the targeted level, hiring drivers had another prominent benefit. Drivers were asked to $\log$ the driving requests that they were unable to perform due to unavailable vehicles or parking spots. They were thus providing information regarding unfulfilled requests, information that is typically unavailable to system operators. As the characteristics of the driver requests follow the patterns observed in the trip transaction data, the percentage of unfulfilled driver requests provide a rough estimate of the global percentage of unfulfilled requests in the system during the experiment days.

During the field experiment, relocators' schedules were adapted such that at least one relocator was available to perform relocations during the active periods of the system. Namely, two staff members were working in overlapping 7-hour shifts spanning from $7 \mathrm{am}$ to $8 \mathrm{pm}$.

Finally, in order to efficiently communicate relocation decisions to the relocators, we designed a web application interface. The application was hosted on a server and accessible for the relocators through their smartphones. Every time the application was queried by a relocator, the corresponding algorithm was providing the best relocation task to perform next, taking into account the relocator's location and the current state of the system.

During the field experiment we have tested four relocation policies, namely, no relocation, the OVOS policy, the Markovian estimation policy and the operator's policy. In addition, during two days of the experiment $(02.10 .2017$, 03.10.2017) we have tested the impact of jockey redistributions. Specifically, a pool of preferred frequent users (jockey users) were offered free trips that originated at target origin stations and ended at target destination stations. These trips were dynamically identified and updated using the algorithms presented in Section 2. The jockeys had access to another web platform where current relevant trips were listed. During the last day of the second field experiment, we also tested the impact of origin-destination shifting. In that case, drivers were advised to shift to stations in walking distance from their origin/destination stations based on resource (vehicle/spot) availabilities. While developing proper algorithms to enhance relocations through jockeys' actions is an interesting research direction, we consider it beyond the scope of this work as it includes additional behavioral and pricing aspects that would have to be further investigated.

The results of the two field experiments are summarized in Table 4, Table 5 and Table 6 . The tables report for each day of the experiment the relocation policy applied, the number of available vehicles in the system, the number of realized demands (in total, by drivers and by users), the amount of redistributions performed (by relocators and by jockeys when applicable) and the percentage of driver requests that were unfulfilled due to vehicle or parking spot shortages. 


\begin{tabular}{|l|c|c|c|c|}
\cline { 2 - 5 } \multicolumn{1}{c|}{} & $\begin{array}{c}\text { Tuesday } \\
27.06 .2017\end{array}$ & $\begin{array}{c}\text { Wednesday } \\
\text { 28.06.2017 }\end{array}$ & $\begin{array}{c}\text { Thursday } \\
29.06 .2017\end{array}$ & $\begin{array}{c}\text { Friday } \\
\text { 28.06.2017 }\end{array}$ \\
\hline Relocation Policy & No Relocation & OVOS & OVOS & Operator \\
\hline Number of vehicles & 50 & 53 & 48 & 48 \\
\hline Daily demands & 100 & 103 & 117 & 105 \\
\hline - realized by users & 63 & 77 & 76 & 69 \\
- realized by drivers & 37 & 26 & 41 & 36 \\
\hline Relocators per shift & 1 & 1 & 1 & 1 \\
\hline Redistributions & - & 28 & 20 & 23 \\
\hline Jockey redistributions & NA & NA & NA & NA \\
\hline Unfulfilled drivers' requests & $29 \%$ & $13 \%$ & $18 \%$ & $31 \%$ \\
\hline
\end{tabular}

Table 4: Results from the first field experiment, June 2017

\begin{tabular}{|l|c|c|c|c|}
\cline { 2 - 5 } \multicolumn{1}{c|}{} & $\begin{array}{c}\text { Tuesday } \\
\text { 26.09.2017 }\end{array}$ & $\begin{array}{c}\text { Wednesday } \\
27.09 .2017\end{array}$ & $\begin{array}{c}\text { Thursday } \\
29.09 .2017\end{array}$ & $\begin{array}{c}\text { Friday } \\
30.09 .2017\end{array}$ \\
\hline Relocation Policy & OVOS & OVOS & Operator & No Relocation \\
\hline Number of vehicles & $40-50$ & $40-50$ & $40-50$ & $40-50$ \\
\hline Daily demands & 108 & 117 & 112 & 114 \\
\hline - realized by users & 68 & 78 & 79 & 83 \\
- realized by drivers & 40 & 39 & 33 & 31 \\
\hline Relocators per shift & 1 & 1 & 1 & - \\
\hline Redistributions & 20 & 26 & 24 & - \\
\hline Jockey redistributions & NA & NA & NA & NA \\
\hline Unfulfilled drivers' requests & $17 \%$ & $26 \%$ & $33 \%$ & $39 \%$ \\
\hline
\end{tabular}

Table 5: Results from the second field experiment, September - October 2017

\begin{tabular}{|l|c|c|c|c|}
\cline { 2 - 5 } \multicolumn{1}{c|}{} & $\begin{array}{c}\text { Monday } \\
02.10 .2017\end{array}$ & $\begin{array}{c}\text { Tuesday } \\
03.10 .2017\end{array}$ & $\begin{array}{c}\text { Wednesday } \\
04.10 .2017\end{array}$ & $\begin{array}{c}\text { Thursday } \\
05.10 .2017\end{array}$ \\
\hline Relocation Policy & OVOS & $\begin{array}{c}\text { Markovian } \\
\text { Policy }\end{array}$ & $\begin{array}{c}\text { Markovian } \\
\text { Policy }\end{array}$ & $\begin{array}{c}\text { Markovian Policy } \\
\text { + OD Shifting }\end{array}$ \\
\hline Number of vehicles & $40-50$ & $40-50$ & $40-50$ & $40-50$ \\
\hline Daily demands & 105 & 121 & 94 & 110 \\
\hline - realized by users & 73 & 81 & 64 & 83 \\
- realized by drivers & 32 & 40 & 30 & 27 \\
\hline Relocators per shift & 1 & 1 & 1 & 1 \\
\hline Redistributions & 14 & 23 & 25 & 13 \\
\hline Jockey redistributions & 8 & 4 & NA & NA \\
\hline Unfulfilled drivers' requests & $31 \%$ & $26 \%$ & $22 \%$ & $14 \%$ \\
\hline
\end{tabular}

Table 6: Results from the second field experiment, September - October 2017 
As can be observed, the target level of 100 demands per day was reached in 11 out of 12 days of the experiments. User demand accounted for about two thirds of the total demand. During the second field experiment, the number of available vehicles varied greatly due to maintenance actions taken in parallel to the experiment.

Most of the documented unfulfilled driver requests were due to vehicle shortages in the origin stations. This can be explained by the relatively low number of vehicles distributed in the system. In addition, the booking process through the app required to reserve a vehicle before reserving a destination spot. As a consequence, some unfulfilled requests attributed to lack of vehicles at the origins may also have been unfulfilled due to lack of available parking spots at the destinations.

On average, the OVOS policy and the Markovian estimation policy performed considerably better than the norelocation policy and the operator policy in terms of the percentage of unfulfilled driver requests. This was obtained with the same number of relocators, performing more or less the same amount of relocations. We note that, unfortunately, during the experiment, the operator's information system was not differentiating between reserved vehicles and reserved spots. In order to apply the Markovian estimation policy, the states of the reserved resources were estimated by extrapolating on-line information and historical data. In some cases, imperfect estimations may have led to non-optimal relocation decisions. Nevertheless, the Markovian estimation policy performed well. We are confident that with the exact information regarding the reserved vehicles and spots, which is typically available, the Markovian estimation policy would have produced even better results. This is shown later in the paper through detailed simulation tests.

During the first field experiment, we also followed the aggregate availability state of the system with regards to the definition criterion of the OVOS policy: 1 vehicle and 1 parking spot available. We present this evolution in Figure 7. The three curves describe the number of stations in each of the following inventory states: at least 1 available vehicle and 1 available spot, no available spot, and no available vehicle. The graph emphasizes the positive impact of any relocation policy on the general state availability of resources in the system compared to no relocation policy. Only in cases where a relocation policy, Operator or OVOS, is implemented, the system is able to reach a high number of stations with available vehicles and spots and maintain them in a relatively good state. Note that the drop of the red curve in the evening of June 30 is because relocators stopped their operation earlier (around 5pm).

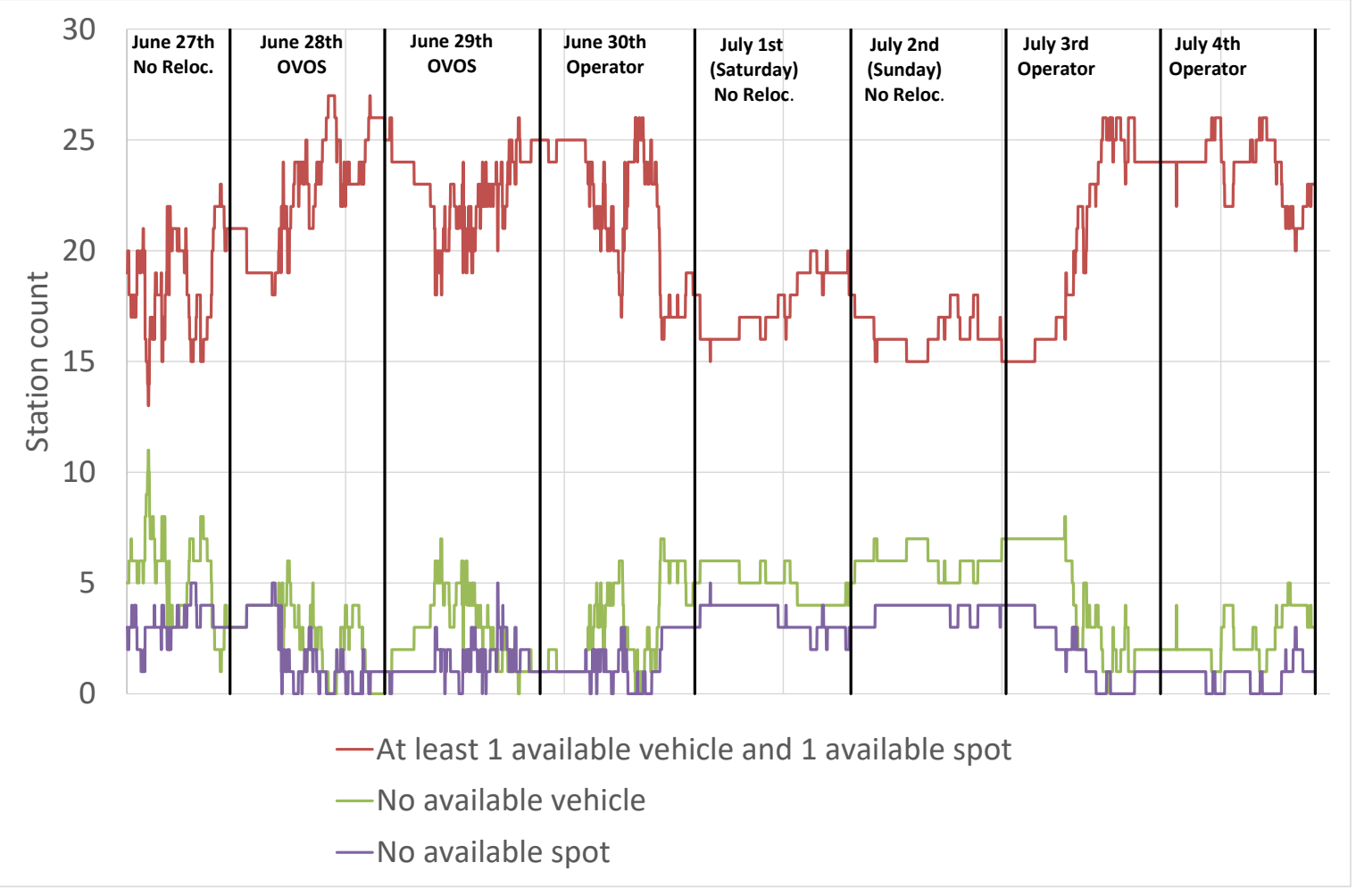

Figure 7: Aggregate station count evolution during the first field test 
The main outcome of the field experiments is that they have demonstrated both the ability to implement the proposed policies in real-world systems. Specifically, they allowed us to test the policies under real-world conditions and evaluate their performance taking into account many factors that cannot always be represented accurately in mathematical or simulation models. However, the significance of the obtained results is limited by the number of days in which the experiments were carried out. To reinforce these results, we have used an event-based simulation framework to test the relocation policies under various system settings, as will be presented in the following section.

\subsection{Simulation}

The event based simulation framework used in our experiments is an updated version of the framework developed by Repoux et al. (2015) and later used by Boyac1 et al. (2017). The reader is referred to these papers for a more detailed description of the simulation framework. In brief, the framework consists of an event-based simulation module communicating with several auxiliary modules. In particular, a relocation decision module implements the relocation policies studied in this paper and is called by the relevant events from the main simulation frame to return decisions. This modularity allows to test different algorithms while keeping the same main frame.

The input of the simulator contains information regarding the network characteristics, the vehicle fleet, the user demand, relocators' working shifts and service regulations. The location of all stations and their capacities were obtained from the operator's information system. Driving times and walking times between stations of the system were derived using GoogleMaps and public transport trip durations were obtained from the public transport service provider of the Grenoble area. The latter two were used to represent the moving time of relocators, taken as the minimum of using public transportation and walking time. Vehicle fleet information contained the number of vehicles distributed in the system and their initial location at the beginning of the simulation. The initial locations of the vehicles were fixed for all simulation runs having the same fleet size.

With respect to the demand, we generated 100 random demand realizations per demand load, based on the patterns exhibited in the trip history transactions. Each realization consists of ten consecutive operating days, as simulating several days consecutively reduces significantly the effect of the initial vehicle distribution on the system's performance. Rentals were drawn from the pool of historical transaction data in the following manner. A random value uniformly drawn between 0 and 1 is assigned to each transaction. Transactions are then sorted ascendingly according to these values and the $n$ first rentals constitute the demand for the first day, $n$ being the number of demands per day considered. The $n$ next transactions correspond to the second day of the seed and so on until the tenth day. As to relocators' shifts, each shift was set to span over the entire demand peak, that is starting from 7am and finishing at 8pm.

The experimental design consists in four levels of daily demand, three fleet sizes and three relocator staff sizes, as summarized in Table 7. In the rest of this section, we refer to each combination of the three as a system configuration. Demand levels range between 100 demands per day and 400 demands per day. The former is the target level set during the field experiments while the latter corresponds to approximately ten daily rentals per vehicle in the real system setting. Thus, the extreme case of 400 demands per day allows us to see how the policies perform in a heavy-loaded case. Investigated fleet size values were selected symmetrically around 60 vehicles, which is half the total number of spots installed in the system. The 40 vehicles and 80 vehicles cases describe situations with a surplus of spots and a surplus of vehicles, respectively.

\begin{tabular}{|l|l|}
\hline Demand per day & $100,200,300,400$ \\
\hline Fleet size & $40,60,80$ \\
\hline Staff size & $1,2,3$ \\
\hline
\end{tabular}

Table 7: System configurations tested in the simulation experiment

The simulation results are presented in Table 8, Table 9 and Table 10 for system configurations using one, two and three relocators, respectively. In each table, the first three columns present the system setting in terms of the daily demand, the number of vehicles and the tested relocation policy. The next five columns represent under each setting the percentage of served requests, the number of relocations performed, the relocators' idle time, moving time and driving time. These values are calculated by averaging over 100 different 10-days simulations corresponding to the 100 demand realizations generated for each demand load. We note that the results presented for the Markovian policies were obtained using a 2-hours prediction horizon. Prediction horizons of 1 and 3 hours were also tested and 
have resulted with very similar performance, demonstrating that the model is not sensitive to reasonable prediction horizons. For all configurations tested, the OVOS and the Markovian policies have resulted with significantly higher percentages of served requests, as compared to the no-relocation policy. This outcome reconfirms the conclusions of many previous studies. Namely, that relocations can increase significantly the quality of service provided to the users of the system. Comparing the OVOS and the Markovian estimation policy demonstrates that accounting for reservation information and historical demand results with an increase of $1.4 \%$ up to $4.7 \%$ in the percentage of served demand. A deeper analysis shows that the improvement is consistent over almost all the demand realizations. In fact, in 24 out of the 36 tested configurations, the Markovian estimation policy performs better than the OVOS policy on all demand seeds. In each of the remaining 12 configurations, a maximum of 3 demand realizations, over the 100 tested, exhibited better results with the OVOS policy than with the Markovian estimation policy. One might argue that the improvement that the Markovian policy offers as compared to OVOS policy is not very high. However, we highlight that the reactive OVOS policy already results with fairly high level of service. In addition, in several configurations, the potential additional improvement is quite small as suggested by the approximated upper bound. The smallest relative improvements correspond to configurations where the percentage of served requests is already close to $95 \%$ or high-demand single-staff configurations, where relocations have only a minor effect. Depending on the configuration, shifting from the OVOS policy to the Markovian policy closes between $13 \%$ to $45 \%$ of the remaining gap towards the approximated upper bound. Recalling that the bound is based on centralistic decision making using full knowledge of the demand, the actual relative improvement is most likely much higher.

Focusing on the relocators' activities, we see that in most configurations, under the OVOS and the Markovian policies, the relocators spend approximately the same time idling. This emphasizes that the improvement is not coming from a higher resource utilization but from the more efficient task selection for the available personnel. Notably, considerably higher idle times are overserved for the OVOS policy only in cases where the daily demand is low (100) and there are two or three relocators. In such cases, the vehicle rotation rate is also low and generally vehicle inventories as well. The chances for the system to be in a good state according to the OVOS policy criteria are higher and the relocators have higher probability to remain idle. On the contrary, the Markovian estimation policy, which estimates the future losses having aggregate knowledge over the demand, may induce inventory shortages in order to fill up or deplete stations where demands are more likely to appear. This results in a higher number of relocation movements in cases where the global performance is already high. We observe also that, in general, driving time proportion is slightly higher in the Markovian estimation policy than in the OVOS policy while the idling time is approximately the same. Relocators spend then more time effectively transferring resources than accessing them, which results in an improved level of service.

With respect to the fleet size, we observe that the configurations with 60 vehicles generally result with better performance as compared to the 40 and 80 vehicle configurations. Interestingly, the configuration with 80 vehicles performs much better than the 40 vehicle case, even though the two cases represent mirroring vehicle/parking spot asymmetry. Moreover, for high daily demands, in some configurations, using 80 vehicles gives the best performance. This outcome aligns with the analytical results of Fricker and Gast (2016) for bike sharing systems, stating that the ideal fleet size is approximately half of the number of parking spots in the system plus the average number of vehicles travelling. Indeed, in the case studied system, under high daily demand, this number is likely to be closer to 80 than to 60.

As for the staff size, a decreasing marginal contribution is observed, namely the contribution of the second and third relocator are smaller than the improvement seen introducing the first relocator. Specifically, for low demand levels, the added value of the second and third relocators are almost insignificant, independently of the policy applied. 


\begin{tabular}{|c|c|c|c|c|c|c|c|}
\hline \multirow[t]{2}{*}{ Demand } & \multirow[t]{2}{*}{ Vehicles } & \multirow[t]{2}{*}{ Policy } & \multirow[t]{2}{*}{$\%$ served } & \multirow{2}{*}{$\begin{array}{c}\text { Relocations } \\
\text { per day }\end{array}$} & \multicolumn{3}{|c|}{ Relocator activity } \\
\hline & & & & & Idle & Move & Drive \\
\hline \multirow{12}{*}{100} & \multirow{4}{*}{40} & No Relocations & $59.5 \%$ & 0.0 & $100.0 \%$ & $0.0 \%$ & $0.0 \%$ \\
\hline & & OVOS & $80.1 \%$ & 31.5 & $2.9 \%$ & $66.6 \%$ & $30.5 \%$ \\
\hline & & Markovian Estimation & $84.7 \%$ & 33.9 & $1.5 \%$ & $63.2 \%$ & $35.3 \%$ \\
\hline & & Approx. UB & $94.7 \%$ & 21.9 & $4.0 \%$ & $50.7 \%$ & $45.3 \%$ \\
\hline & \multirow{4}{*}{60} & No Relocations & $68.8 \%$ & 0.0 & $100.0 \%$ & $0.0 \%$ & $0.0 \%$ \\
\hline & & OVOS & $88.5 \%$ & 24.8 & $3.0 \%$ & $67.2 \%$ & $29.8 \%$ \\
\hline & & Markovian Estimation & $92.3 \%$ & 35.3 & $1.6 \%$ & $63.7 \%$ & $34.7 \%$ \\
\hline & & Approx. UB & $97.1 \%$ & 19.5 & $5.2 \%$ & $52.7 \%$ & $42.1 \%$ \\
\hline & \multirow{4}{*}{80} & No Relocations & $68.4 \%$ & 0.0 & $100.0 \%$ & $0.0 \%$ & $0.0 \%$ \\
\hline & & OVOS & $87.7 \%$ & 30.1 & $2.4 \%$ & $70.3 \%$ & $27.3 \%$ \\
\hline & & Markovian Estimation & $90.2 \%$ & 33.9 & $1.6 \%$ & $67.2 \%$ & $31.2 \%$ \\
\hline & & Approx. UB & $96.4 \%$ & 20.7 & $4.4 \%$ & $51.0 \%$ & $44.6 \%$ \\
\hline \multirow{12}{*}{200} & \multirow{4}{*}{40} & No Relocations & $55.3 \%$ & 0.0 & $100.0 \%$ & $0.0 \%$ & $0.0 \%$ \\
\hline & & OVOS & $66.8 \%$ & 32.7 & $1.9 \%$ & $72.8 \%$ & $25.2 \%$ \\
\hline & & Markovian Estimation & $70.6 \%$ & 32.6 & $1.6 \%$ & $66.0 \%$ & $32.5 \%$ \\
\hline & & Approx. UB & $83.2 \%$ & 22.6 & $3.6 \%$ & $50.5 \%$ & $45.8 \%$ \\
\hline & \multirow{4}{*}{60} & No Relocations & $65.5 \%$ & 0.0 & $100.0 \%$ & $0.0 \%$ & $0.0 \%$ \\
\hline & & OVOS & $77.3 \%$ & 26.4 & $2.0 \%$ & $69.4 \%$ & $28.6 \%$ \\
\hline & & Markovian Estimation & $80.9 \%$ & 35.3 & $1.6 \%$ & $65.5 \%$ & $32.9 \%$ \\
\hline & & Approx. UB & $89.5 \%$ & 22.9 & $3.4 \%$ & $49.9 \%$ & $46.8 \%$ \\
\hline & \multirow{4}{*}{80} & No Relocations & $66.1 \%$ & 0.0 & $100.0 \%$ & $0.0 \%$ & $0.0 \%$ \\
\hline & & OVOS & $78.6 \%$ & 33.5 & $1.7 \%$ & $66.3 \%$ & $32.0 \%$ \\
\hline & & Markovian Estimation & $80.7 \%$ & 37.1 & $1.5 \%$ & $65.4 \%$ & $33.1 \%$ \\
\hline & & Approx. UB & $88.1 \%$ & 23.3 & $3.1 \%$ & $49.1 \%$ & $47.8 \%$ \\
\hline \multirow{12}{*}{300} & \multirow{4}{*}{40} & No Relocations & $51.4 \%$ & 0.0 & $100.0 \%$ & $0.0 \%$ & $0.0 \%$ \\
\hline & & OVOS & $58.7 \%$ & 32.4 & $1.8 \%$ & $74.9 \%$ & $23.2 \%$ \\
\hline & & Markovian Estimation & $61.6 \%$ & 30.9 & $1.6 \%$ & $67.1 \%$ & $31.3 \%$ \\
\hline & & Approx. UB & $75.0 \%$ & 22.5 & $3.8 \%$ & $51.0 \%$ & $45.2 \%$ \\
\hline & \multirow{4}{*}{60} & No Relocations & $61.7 \%$ & 0.0 & $100.0 \%$ & $0.0 \%$ & $0.0 \%$ \\
\hline & & OVOS & $69.8 \%$ & 28.9 & $1.6 \%$ & $71.3 \%$ & $27.1 \%$ \\
\hline & & Markovian Estimation & $72.6 \%$ & 34.8 & $1.5 \%$ & $66.4 \%$ & $32.1 \%$ \\
\hline & & Approx. UB & $82.6 \%$ & 23.2 & $3.2 \%$ & $50.0 \%$ & $46.8 \%$ \\
\hline & \multirow{4}{*}{80} & No Relocations & $62.9 \%$ & 0.0 & $100.0 \%$ & $0.0 \%$ & $0.0 \%$ \\
\hline & & OVOS & $71.8 \%$ & 35.3 & $1.6 \%$ & $64.0 \%$ & $34.4 \%$ \\
\hline & & Markovian Estimation & $73.8 \%$ & 37.4 & $1.4 \%$ & $65.0 \%$ & $33.5 \%$ \\
\hline & & Approx. UB & $81.7 \%$ & 23.5 & $2.9 \%$ & $49.4 \%$ & $47.6 \%$ \\
\hline \multirow{12}{*}{400} & \multirow{4}{*}{40} & No Relocations & $47.8 \%$ & 0.0 & $100.0 \%$ & $0.0 \%$ & $0.0 \%$ \\
\hline & & OVOS & $52.8 \%$ & 32.3 & $1.8 \%$ & $75.8 \%$ & $22.4 \%$ \\
\hline & & Markovian Estimation & $55.0 \%$ & 29.4 & $1.7 \%$ & $67.6 \%$ & $30.8 \%$ \\
\hline & & Approx. UB & $69.4 \%$ & 22.4 & $3.7 \%$ & $51.6 \%$ & $44.7 \%$ \\
\hline & & No Relocations & $58.0 \%$ & 0.0 & $100.0 \%$ & $0.0 \%$ & $0.0 \%$ \\
\hline & 60 & OVOS & $64.1 \%$ & 30.5 & $1.5 \%$ & $72.2 \%$ & $26.2 \%$ \\
\hline & & Markovian Estimation & $66.2 \%$ & 33.4 & $1.5 \%$ & $66.7 \%$ & $31.7 \%$ \\
\hline & & Approx. UB & $77.7 \%$ & 23.2 & $3.0 \%$ & $50.6 \%$ & $46.4 \%$ \\
\hline & & No Relocations & $59.7 \%$ & 0.0 & $100.0 \%$ & $0.0 \%$ & $0.0 \%$ \\
\hline & 80 & OVOS & $66.6 \%$ & 36.3 & $1.7 \%$ & $63.1 \%$ & $35.2 \%$ \\
\hline & & Markovian Estimation & $68.1 \%$ & 36.5 & $1.5 \%$ & $65.2 \%$ & $33.4 \%$ \\
\hline & & Approx. UB & $77.5 \%$ & 23.6 & $2.6 \%$ & $50.0 \%$ & $47.4 \%$ \\
\hline
\end{tabular}

Table 8: Simulation results with one personnel 


\begin{tabular}{|c|c|c|c|c|c|c|c|}
\hline \multirow[t]{2}{*}{ Demand } & \multirow[t]{2}{*}{ Vehicles } & \multirow[t]{2}{*}{ Policy } & \multirow[t]{2}{*}{$\%$ served } & \multirow{2}{*}{$\begin{array}{c}\text { Relocations } \\
\text { per day }\end{array}$} & \multicolumn{3}{|c|}{ Relocator activity } \\
\hline & & & & & Idle & Move & Drive \\
\hline \multirow{12}{*}{100} & \multirow{4}{*}{40} & No Relocations & $59.5 \%$ & 0.0 & $100.0 \%$ & $0.0 \%$ & $0.0 \%$ \\
\hline & & OVOS & $86.1 \%$ & 52.6 & $15.0 \%$ & $53.5 \%$ & $31.4 \%$ \\
\hline & & Markovian Estimation & $89.7 \%$ & 62.4 & $2.8 \%$ & $60.8 \%$ & $36.4 \%$ \\
\hline & & Approx. UB & $97.8 \%$ & 35.0 & $5.9 \%$ & $54.8 \%$ & $39.2 \%$ \\
\hline & \multirow{4}{*}{60} & No Relocations & $68.8 \%$ & 0.0 & $100.0 \%$ & $0.0 \%$ & $0.0 \%$ \\
\hline & & OVOS & $92.9 \%$ & 46.0 & $19.3 \%$ & $55.3 \%$ & $25.4 \%$ \\
\hline & & Markovian Estimation & $95.6 \%$ & 64.7 & $2.2 \%$ & $61.1 \%$ & $36.7 \%$ \\
\hline & & Approx. UB & $98.2 \%$ & 33.3 & $5.7 \%$ & $55.9 \%$ & $38.4 \%$ \\
\hline & \multirow{4}{*}{80} & No Relocations & $68.4 \%$ & 0.0 & $100.0 \%$ & $0.0 \%$ & $0.0 \%$ \\
\hline & & OVOS & $92.2 \%$ & 51.3 & $20.7 \%$ & $54.6 \%$ & $24.7 \%$ \\
\hline & & Markovian Estimation & $94.0 \%$ & 63.0 & $2.0 \%$ & $63.9 \%$ & $34.1 \%$ \\
\hline & & Approx. UB & $98.0 \%$ & 34.3 & $5.4 \%$ & $53.8 \%$ & $40.8 \%$ \\
\hline \multirow{12}{*}{200} & \multirow{4}{*}{40} & No Relocations & $55.3 \%$ & 0.0 & $100.0 \%$ & $0.0 \%$ & $0.0 \%$ \\
\hline & & OVOS & $71.6 \%$ & 64.4 & $2.3 \%$ & $67.7 \%$ & $30.0 \%$ \\
\hline & & Markovian Estimation & $75.9 \%$ & 59.3 & $1.9 \%$ & $64.9 \%$ & $33.2 \%$ \\
\hline & & Approx. UB & $92.3 \%$ & 43.7 & $3.6 \%$ & $52.2 \%$ & $44.2 \%$ \\
\hline & \multirow{4}{*}{60} & No Relocations & $65.5 \%$ & 0.0 & $100.0 \%$ & $0.0 \%$ & $0.0 \%$ \\
\hline & & OVOS & $81.8 \%$ & 49.0 & $3.7 \%$ & $66.0 \%$ & $30.3 \%$ \\
\hline & & Markovian Estimation & $86.5 \%$ & 65.1 & $1.6 \%$ & $64.2 \%$ & $34.2 \%$ \\
\hline & & Approx. UB & $95.7 \%$ & 40.7 & $4.1 \%$ & $53.2 \%$ & $42.7 \%$ \\
\hline & \multirow{4}{*}{80} & No Relocations & $66.1 \%$ & 0.0 & $100.0 \%$ & $0.0 \%$ & $0.0 \%$ \\
\hline & & OVOS & $82.8 \%$ & 59.1 & $3.2 \%$ & $67.9 \%$ & $28.9 \%$ \\
\hline & & Markovian Estimation & $85.9 \%$ & 67.0 & $1.6 \%$ & $64.8 \%$ & $33.6 \%$ \\
\hline & & Approx. UB & $95.3 \%$ & 41.6 & $3.7 \%$ & $52.5 \%$ & $43.8 \%$ \\
\hline \multirow{12}{*}{300} & \multirow{4}{*}{40} & No Relocations & $51.4 \%$ & 0.0 & $100.0 \%$ & $0.0 \%$ & $0.0 \%$ \\
\hline & & OVOS & $62.4 \%$ & 67.3 & $2.0 \%$ & $71.2 \%$ & $26.7 \%$ \\
\hline & & Markovian Estimation & $65.8 \%$ & 56.3 & $1.9 \%$ & $66.1 \%$ & $32.1 \%$ \\
\hline & & Approx. UB & $81.8 \%$ & 44.9 & $3.5 \%$ & $52.0 \%$ & $44.5 \%$ \\
\hline & \multirow{4}{*}{60} & No Relocations & $61.7 \%$ & 0.0 & $100.0 \%$ & $0.0 \%$ & $0.0 \%$ \\
\hline & & OVOS & $73.4 \%$ & 50.7 & $2.4 \%$ & $68.1 \%$ & $29.5 \%$ \\
\hline & & Markovian Estimation & $77.6 \%$ & 62.9 & $1.6 \%$ & $65.4 \%$ & $33.0 \%$ \\
\hline & & Approx. UB & $89.5 \%$ & 45.3 & $3.0 \%$ & $51.9 \%$ & $45.1 \%$ \\
\hline & \multirow{4}{*}{80} & No Relocations & $62.9 \%$ & 0.0 & $100.0 \%$ & $0.0 \%$ & $0.0 \%$ \\
\hline & & OVOS & $75.4 \%$ & 60.7 & $1.8 \%$ & $67.5 \%$ & $30.7 \%$ \\
\hline & & Markovian Estimation & $78.5 \%$ & 67.1 & $1.5 \%$ & $64.6 \%$ & $33.8 \%$ \\
\hline & & Approx. UB & $89.2 \%$ & 45.9 & $2.8 \%$ & $51.1 \%$ & $46.1 \%$ \\
\hline \multirow{12}{*}{400} & \multirow{4}{*}{40} & No Relocations & $47.8 \%$ & 0.0 & $100.0 \%$ & $0.0 \%$ & $0.0 \%$ \\
\hline & & OVOS & $55.6 \%$ & 67.2 & $2.2 \%$ & $72.8 \%$ & $24.9 \%$ \\
\hline & & Markovian Estimation & $58.2 \%$ & 53.7 & $1.9 \%$ & $66.5 \%$ & $31.5 \%$ \\
\hline & & Approx. UB & $74.7 \%$ & 44.9 & $3.2 \%$ & $52.6 \%$ & $44.2 \%$ \\
\hline & & No Relocations & $58.0 \%$ & 0.0 & $100.0 \%$ & $0.0 \%$ & $0.0 \%$ \\
\hline & 60 & OVOS & $67.0 \%$ & 53.5 & $1.9 \%$ & $69.7 \%$ & $28.4 \%$ \\
\hline & & Markovian Estimation & $70.4 \%$ & 60.6 & $1.7 \%$ & $65.9 \%$ & $32.4 \%$ \\
\hline & & Approx. UB & $83.1 \%$ & 46.8 & $2.5 \%$ & $51.5 \%$ & $46.1 \%$ \\
\hline & & No Relocations & $59.7 \%$ & 0.0 & $100.0 \%$ & $0.0 \%$ & $0.0 \%$ \\
\hline & 80 & OVOS & $69.6 \%$ & 61.5 & $1.7 \%$ & $65.8 \%$ & $32.5 \%$ \\
\hline & & Markovian Estimation & $72.3 \%$ & 65.3 & $1.6 \%$ & $64.6 \%$ & $33.8 \%$ \\
\hline & & Approx. UB & $83.4 \%$ & 47.6 & $2.4 \%$ & $50.3 \%$ & $47.3 \%$ \\
\hline
\end{tabular}

Table 9: Simulation results with two personnel 


\begin{tabular}{|c|c|c|c|c|c|c|c|}
\hline \multirow[t]{2}{*}{ Demand } & \multirow[t]{2}{*}{ Vehicles } & \multirow[t]{2}{*}{ Policy } & \multirow[t]{2}{*}{$\%$ served } & \multirow{2}{*}{$\begin{array}{c}\text { Relocations } \\
\text { per day }\end{array}$} & \multicolumn{3}{|c|}{ Relocator activity } \\
\hline & & & & & Idle & Move & Drive \\
\hline \multirow{12}{*}{100} & \multirow{4}{*}{40} & No Relocations & $59.5 \%$ & 0.0 & $100.0 \%$ & $0.0 \%$ & $0.0 \%$ \\
\hline & & OVOS & $88.0 \%$ & 61.9 & $32.0 \%$ & $41.8 \%$ & $26.2 \%$ \\
\hline & & Markovian Estimation & $91.2 \%$ & 86.1 & $9.7 \%$ & $55.2 \%$ & $35.1 \%$ \\
\hline & & Approx. UB & $97.9 \%$ & 47.5 & $6.5 \%$ & $56.1 \%$ & $37.4 \%$ \\
\hline & \multirow{4}{*}{60} & No Relocations & $68.8 \%$ & 0.0 & $100.0 \%$ & $0.0 \%$ & $0.0 \%$ \\
\hline & & OVOS & $94.1 \%$ & 55.5 & $39.4 \%$ & $41.2 \%$ & $19.5 \%$ \\
\hline & & Markovian Estimation & $96.4 \%$ & 89.7 & $6.8 \%$ & $56.4 \%$ & $36.8 \%$ \\
\hline & & Approx. UB & $98.2 \%$ & 47.0 & $6.1 \%$ & $56.6 \%$ & $37.3 \%$ \\
\hline & \multirow{4}{*}{80} & No Relocations & $68.4 \%$ & 0.0 & $100.0 \%$ & $0.0 \%$ & $0.0 \%$ \\
\hline & & OVOS & $93.5 \%$ & 59.2 & $40.7 \%$ & $40.0 \%$ & $19.3 \%$ \\
\hline & & Markovian Estimation & $94.8 \%$ & 87.4 & $4.8 \%$ & $60.4 \%$ & $34.8 \%$ \\
\hline & & Approx. UB & $98.1 \%$ & 47.9 & $5.7 \%$ & $54.7 \%$ & $39.6 \%$ \\
\hline \multirow{12}{*}{200} & \multirow{4}{*}{40} & No Relocations & $55.3 \%$ & 0.0 & $100.0 \%$ & $0.0 \%$ & $0.0 \%$ \\
\hline & & OVOS & $73.8 \%$ & 84.2 & $5.1 \%$ & $63.2 \%$ & $31.7 \%$ \\
\hline & & Markovian Estimation & $77.8 \%$ & 82.7 & $4.6 \%$ & $62.2 \%$ & $33.2 \%$ \\
\hline & & Approx. UB & $95.7 \%$ & 57.9 & $4.9 \%$ & $53.7 \%$ & $41.4 \%$ \\
\hline & \multirow{4}{*}{60} & No Relocations & $65.5 \%$ & 0.0 & $100.0 \%$ & $0.0 \%$ & $0.0 \%$ \\
\hline & & OVOS & $84.5 \%$ & 74.1 & $11.7 \%$ & $59.4 \%$ & $28.9 \%$ \\
\hline & & Markovian Estimation & $88.6 \%$ & 92.8 & $2.0 \%$ & $62.5 \%$ & $35.5 \%$ \\
\hline & & Approx. UB & $96.7 \%$ & 54.0 & $5.3 \%$ & $54.6 \%$ & $40.1 \%$ \\
\hline & \multirow{4}{*}{80} & No Relocations & $66.1 \%$ & 0.0 & $100.0 \%$ & $0.0 \%$ & $0.0 \%$ \\
\hline & & OVOS & $85.2 \%$ & 82.7 & $11.1 \%$ & $61.4 \%$ & $27.5 \%$ \\
\hline & & Markovian Estimation & $88.0 \%$ & 95.6 & $1.9 \%$ & $63.2 \%$ & $34.9 \%$ \\
\hline & & Approx. UB & $96.6 \%$ & 55.2 & $4.9 \%$ & $53.5 \%$ & $41.6 \%$ \\
\hline \multirow{12}{*}{300} & \multirow{4}{*}{40} & No Relocations & $51.4 \%$ & 0.0 & $100.0 \%$ & $0.0 \%$ & $0.0 \%$ \\
\hline & & OVOS & $64.0 \%$ & 91.5 & $3.4 \%$ & $68.4 \%$ & $28.2 \%$ \\
\hline & & Markovian Estimation & $67.4 \%$ & 79.2 & $3.8 \%$ & $64.1 \%$ & $32.1 \%$ \\
\hline & & Approx. UB & $86.9 \%$ & 65.1 & $3.5 \%$ & $53.3 \%$ & $43.2 \%$ \\
\hline & \multirow{4}{*}{60} & No Relocations & $61.7 \%$ & 0.0 & $100.0 \%$ & $0.0 \%$ & $0.0 \%$ \\
\hline & & OVOS & $75.7 \%$ & 76.2 & $4.3 \%$ & $64.6 \%$ & $31.0 \%$ \\
\hline & & Markovian Estimation & $80.2 \%$ & 89.4 & $1.7 \%$ & $64.3 \%$ & $34.0 \%$ \\
\hline & & Approx. UB & $93.3 \%$ & 61.6 & $3.7 \%$ & $53.8 \%$ & $42.5 \%$ \\
\hline & \multirow{4}{*}{80} & No Relocations & $62.9 \%$ & 0.0 & $100.0 \%$ & $0.0 \%$ & $0.0 \%$ \\
\hline & & OVOS & $77.5 \%$ & 86.2 & $3.4 \%$ & $67.1 \%$ & $29.6 \%$ \\
\hline & & Markovian Estimation & $81.0 \%$ & 95.7 & $1.6 \%$ & $63.7 \%$ & $34.7 \%$ \\
\hline & & Approx. UB & $93.1 \%$ & 61.7 & $3.4 \%$ & $53.6 \%$ & $43.0 \%$ \\
\hline \multirow{12}{*}{400} & \multirow{4}{*}{40} & No Relocations & $47.8 \%$ & 0.0 & $100.0 \%$ & $0.0 \%$ & $0.0 \%$ \\
\hline & & OVOS & $56.8 \%$ & 93.3 & $4.2 \%$ & $70.1 \%$ & $25.7 \%$ \\
\hline & & Markovian Estimation & $59.5 \%$ & 75.8 & $3.8 \%$ & $64.6 \%$ & $31.6 \%$ \\
\hline & & Approx. UB & $78.8 \%$ & 66.2 & $3.3 \%$ & $53.4 \%$ & $43.3 \%$ \\
\hline & & No Relocations & $58.0 \%$ & 0.0 & $100.0 \%$ & $0.0 \%$ & $0.0 \%$ \\
\hline & 60 & OVOS & $68.8 \%$ & 78.3 & $2.7 \%$ & $66.6 \%$ & $30.7 \%$ \\
\hline & & Markovian Estimation & $72.7 \%$ & 86.1 & $1.7 \%$ & $65.2 \%$ & $33.1 \%$ \\
\hline & & Approx. UB & $87.4 \%$ & 67.4 & $2.7 \%$ & $53.2 \%$ & $44.1 \%$ \\
\hline & & No Relocations & $59.7 \%$ & 0.0 & $100.0 \%$ & $0.0 \%$ & $0.0 \%$ \\
\hline & 80 & OVOS & $71.3 \%$ & 85.6 & $2.0 \%$ & $67.2 \%$ & $30.8 \%$ \\
\hline & & Markovian Estimation & $74.7 \%$ & 92.8 & $1.6 \%$ & $64.0 \%$ & $34.4 \%$ \\
\hline & & Approx. UB & $88.0 \%$ & 67.3 & $2.6 \%$ & $52.8 \%$ & $44.6 \%$ \\
\hline
\end{tabular}

Table 10: Simulation results with three personnel 
A higher number of relocations leads to a higher number of customers served at the expense of the relocators' salaries and relocations' driving costs. This is a first-order effect highlighting the direct impact of relocations on the level of service. Besides, there exists a second-order effect related to customers' loyalty in highly reliable systems. By performing relocations, the carsharing operator gains demand both in the short term, by serving the demand on the specific day, and in the long term, by building trust and encouraging customers to use the system more regularly. However, this second-order effect cannot be assessed in the scope of this paper. To complete the discussion, we provide hereafter in Tables 11, 12 and 13 a small cost-benefit analysis for the 200 demands per day case as to see how the extra revenue received from the extra demands served covers relocators' expenses. Estimations for various costs involved are taken from Boyac1 et al. (2015) with a fixed vehicle cost of $20 €$ per day, a cost per kilometer travelled of $0.01 €$ per kilometer and a relocator salary cost of $18 €$ per hour.

\begin{tabular}{|c|c|c|c|c|c|c|c|c|}
\hline Demand & Vehicles & Policy & \% served & $\begin{array}{c}\text { Relocator } \\
\text { cost }\end{array}$ & $\begin{array}{l}\text { Vehicle } \\
\text { cost }\end{array}$ & $\begin{array}{c}\text { Distance } \\
\text { cost }\end{array}$ & $\begin{array}{c}\text { Revenue } \\
(1 € / 15 \mathrm{~min})\end{array}$ & $\begin{array}{c}\text { Revenue } \\
(3.3 € / 15 \mathrm{~min})\end{array}$ \\
\hline \multirow{9}{*}{200} & \multirow{3}{*}{40} & No Relocations & $55.2 \%$ & $0.0 €$ & $800.0 €$ & $6.4 €$ & $539.3 €$ & $1779.5 €$ \\
\hline & & OVOS & $66.7 \%$ & $216.0 €$ & $800.0 €$ & $8.1 €$ & $656.0 €$ & $2164.7 €$ \\
\hline & & Markovian Estimation & $70.7 \%$ & $216.0 €$ & $800.0 €$ & $8.6 €$ & $696.0 €$ & $2296.7 €$ \\
\hline & \multirow{3}{*}{60} & No Relocations & $65.6 \%$ & $0.0 €$ & $1200.0 €$ & $7.7 €$ & $656.4 €$ & $2166.0 €$ \\
\hline & & OVOS & $77.4 \%$ & $216.0 €$ & $1200.0 €$ & $9.5 €$ & $771.6 €$ & $2546.3 €$ \\
\hline & & Markovian Estimation & $80.9 \%$ & $216.0 €$ & $1200.0 €$ & $9.9 €$ & $804.9 €$ & $2656.1 €$ \\
\hline & \multirow{3}{*}{80} & No Relocations & $66.2 \%$ & $0.0 €$ & $1600.0 €$ & $8.1 €$ & $690.2 €$ & $2277.6 €$ \\
\hline & & OVOS & $78.7 \%$ & $216.0 €$ & $1600.0 €$ & $9.8 €$ & $802.7 €$ & $2649.0 €$ \\
\hline & & Markovian Estimation & $80.7 \%$ & $216.0 €$ & $1600.0 €$ & $10.0 €$ & $821.0 €$ & $2709.4 €$ \\
\hline
\end{tabular}

Table 11: Simulation results with one personnel

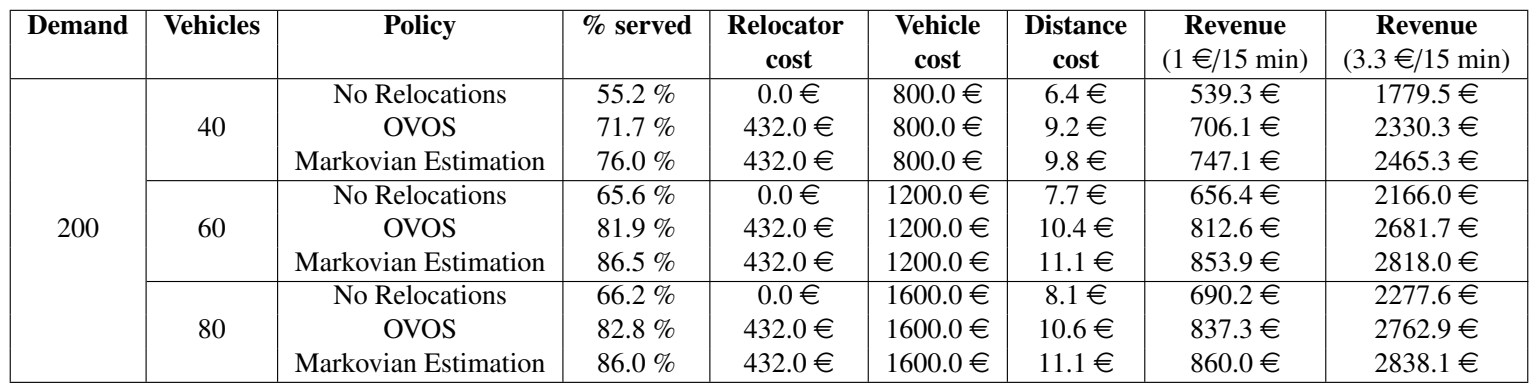

Table 12: Simulation results with two personnel

\begin{tabular}{|c|c|c|c|c|c|c|c|c|}
\hline Demand & Vehicles & Policy & $\%$ served & $\begin{array}{c}\text { Relocator } \\
\text { cost }\end{array}$ & $\begin{array}{c}\text { Vehicle } \\
\text { cost }\end{array}$ & $\begin{array}{l}\text { Distance } \\
\text { cost }\end{array}$ & $\begin{array}{c}\text { Revenue } \\
(1 € / 15 \mathrm{~min})\end{array}$ & $\begin{array}{c}\text { Revenue } \\
(3.3 € / 15 \mathrm{~min})\end{array}$ \\
\hline \multirow{7}{*}{200} & \multirow[b]{2}{*}{40} & No Relocations & $55.2 \%$ & $0.0 €$ & $800.0 €$ & $6.4 €$ & $539.3 €$ & $1779.5 €$ \\
\hline & & OVOS & $73.8 \%$ & $648.0 €$ & $800.0 €$ & $9.9 €$ & $725.5 €$ & $2394.3 €$ \\
\hline & \multirow{3}{*}{60} & No Relocations & $65.6 \%$ & $0.0 €$ & $1200.0 €$ & $7.7 €$ & $656.4 €$ & $2166.0 €$ \\
\hline & & OVOS & $84.4 \%$ & $648.0 €$ & $1200.0 €$ & $11.0 €$ & $835.9 €$ & $2758.5 €$ \\
\hline & & Markovian Estimation & $88.6 \%$ & $648.0 €$ & $1200.0 €$ & $11.9 €$ & $872.2 €$ & $2878.2 €$ \\
\hline & \multirow[t]{2}{*}{80} & OVOS & $85.2 \%$ & $648.0 €$ & $1600.0 €$ & $11.2 €$ & $856.5 €$ & $2826.5 €$ \\
\hline & & Markovian Estimation & $88.1 \%$ & $648.0 €$ & $1600.0 €$ & $11.9 €$ & $877.1 €$ & $2894.4 €$ \\
\hline
\end{tabular}

Table 13: Simulation results with three personnel

Driving distance cost is negligible compared to other important costs, especially the relocators' salary cost. Strategic and tactical investment costs may be disregarded here as they are the same in all studied cases. Table 11 shows that, in the one relocator case and 60 vehicles in operation, extra revenue only covers $72 \%$ of the additional costs due to the relocator's work according to the Grenoble pricing. Nevertheless, comparable France-based carsharing systems offer 
prices at least three times higher than the ones proposed in Grenoble. If the prices of CiteLib by Ha:mo were aligned to the one of Bluely (equiv. $3.3 €$ per quarter of an hour), the Lyon system, the coverage percentages would rise up to $235 \%, 158 \%$ and $115 \%$ for 60 vehicles in operation and respectively one, two and three relocators working. In such conditions, it becomes highly beneficial for the operator to hire relocators while providing a high level of service to the customers, taking advantage of both first and second-order effects of relocation operations.

\section{Conclusion and future research}

In this study, we introduced a dynamic proactive relocation algorithm for carsharing systems implementing a complete journey reservation regulation. The Markovian model used in the algorithm takes into account reservation information as well as historical transaction patterns to estimate quantitatively the expected demand losses in the near-future at every station in the system. The main key performance indicator used to evaluate the algorithm was the number of served requests. We compared the Markovian algorithm to a dynamic reactive inventory-based policy (OVOS), to a static centralistic full-knowledge model and to a benchmark no-relocation case. The results of two field experiments conducted in collaboration with the Grenoble carsharing system and the results of an extensive simulation experiment highlighted the superiority of the Markovian policy.

The field experiments have highlighted challenges unmet in computational studies and therefore, further linked research and practice towards the implementation of efficient relocation algorithms. During the experiments, demand load was artificially increased using hired drivers. The additional data collected by the hired drivers allowed us to reveal information that is typically not available to system operators, for example, the number of unfulfilled service requests. These experiments underlined the positive effect of the two proposed dynamic relocations as compared to not performing relocations and to the relocation policy used by the operators of the system.

The simulation experiments have reinforced the results obtained in the field and allowed us to perform a deeper comparison between the OVOS policy and the Markovian policy. Specifically, we exhibited that the Markovian policy results with superior performance even though relocators' activities were distributed similarly under both policies. This indicated that the improved performance was mainly obtained due to smarter dynamic relocation decisions. Also, the results from the approximated upper bound model (with perfect knowledge of future demand) demonstrate that the further improvements obtained with any complex dynamic relocation policy are rather limited. In addition, the simulation results have once more highlighted the strong effect that fleet sizing may have on operational performance, impact that is only partially mitigated by the introduction of relocations. The interaction between all parameters and settings in carsharing is complex and highly non-linear. It re-emphasizes the importance for any practitioner to identify the most effective elements (namely fleet size, station capacities, rental rules) as well as the ones specific to the system's environment and demand.

Through this study, we have first shown that a better informed carsharing system provides a higher level of service, i.e. serves more demands. We have proposed a model that takes advantage of the aggregated historical data and current system information. Using this partial and incomplete information to smartly plan for relocations, benefits the system and its customers. The approximate upper bound model further extends the comparison by evaluating the level of service in a full extensive information case. This incentivizes practitioners to conceive systems where information is easily retrievable and usable for the future improvements. Second, this study also shows the importance of basic meaningful policies through the example of OVOS. Although OVOS does not incorporate any proactive features, it still does performs well and close to the Markovian Estimation level of service. It was also shown in the field experiments that it outperformed the operator's previous strategy with its very simple set of rules. A well-designed basic policy is also usually easier to set and it is easier and faster to spot incoherences, which is crucial for day-to-day operations. Third, this work demonstrates that coherent staff-based relocation policies have an important impact, especially regarding the effect of the first relocator. Additional relocators only have a limited impact. Although staff-based relocation improves the system's level of service, it might still not be sufficient to keep the customers in the long term. To further increase the level of service, operators may explore other management and rental models. Some of them might require a partial restriction of the customer's freedom by asking, for instance, him/her to give a return time window. Therefore, a system operator has to find the best trade-off between the flexibility given to the users, the means at his/her disposal and the achievable level of service through the rules he/she sets for rental.

As for future research, the presented model may then be used to dynamically redirect users to rental stations which would be more beneficial in terms of expected demand losses. Such a feature is beyond the scope of this paper but the 
Markovian policy can be adapted to fit into incentive procedures that would direct users to reserve at the current best stations with respect to expected future loss. One step further, both dynamic relocation and incentive procedures could be combined to further improve the performance of the system.

Besides, the two dynamic relocation policies presented in this study currently suppose that the complete journey reservation regulations imposed to users are also imposed on the relocators. In particular, relocators are required to reserve vehicles and parking spots before starting their relocation journeys. In some scenarios, this may lead to cases where rejections occur because the current resources are reserved by relocators. Furthermore, by applying complete journey reservation to relocators, two consecutive tasks are decided upon simultaneously, namely, a movement and a relocation. By relaxing the reservation requirement for the relocators, we may introduce more flexibility, allowing a more frequent reevaluation of relocation decisions and adaptation according to the state of the system.

\section{Acknowledgements}

The authors are thankful to the Cité Lib by Ha:mo Grenoble Team for their help, availability and valuable information they provided during the course of this study and especially for their involvement during the field experiments. Special thanks are extended to Dr. El Chammas, Dr. Zgheib and Mr. Kitaoka.

\section{References}

Angelopoulos, A., Gavalas, D., Konstantopoulos, C., Kypriadis, D., Pantziou, G., 2018. Incentivized vehicle relocation in vehicle sharing systems Transportation Research Part C: Emerging Technologies 97, 175-193. http://dx.doi.org/10.1016/j.trc.2018.10.016.

Barth, M., Todd, M., Xue, L., 1999. Simulation model performance analysis of a multiple station shared vehicle system. Transport Research Part C: Emerging Technologies 7, 237-259. http://dx.doi.org/10.1016/S0968-090X(99)00021-2.

Barth, M., Todd, M., Xue, L., 2004. User-based vehicle relocation techniques for multiple-station shared-use vehicle systems. Transportation Research Record: Journal of the Transportation Research Board 1887, 137-144, https://pdfs.semanticscholar.org/f568/ 01840417cee2ad40fd9ac32e9c7239d70770.pdf.

Becker, H., Ciari, F., Axhausen, K. W., 2017a. Comparing car-sharing schemes in switzerland: User groups and usage patterns. Transportation Research Part A: Policy and Practice 97, 17-29. http://dx.doi.org/10.1016/j.tra.2017.01.004.

Becker, H., Ciari, F., Axhausen, K. W., 2017b. Modeling free-floating car-sharing use in Switzerland: A spatial regression and conditional logit approach. Transportation Research Part C: Emerging Technologies 81, 286-299. http://dx.doi.org/10.1016/j.trc.2017.06.008.

Becker, H., Loder, A., Schmid, B., Axhausen, K. W., 2017c. Modeling car-sharing membership as a mobility tool: a multivariate probit approach with latent variables. Travel Behaviour and Society 8, 26-36. http://dx.doi.org/10.1016/j.tbs.2017.04.006.

Boyacı, B., Zografos, K. G., Geroliminis, N., 2015. An optimization framework for the development of efficient one-way car-sharing systems. European Journal of Operational Research 240 (3), 718-733. http://dx.doi.org/10.1016/j.ejor.2014.07.020.

Boyac1, B., Zografos, K. G., Geroliminis, N., 2017. An integrated optimization-simulation framework for vehicle and personnel relocations of electric carsharing systems with reservations. Transportation Research Part B: Methodological 95, 214-237. http://dx.doi.org/10.1016/j.trb.2016.10.007.

Brandstätter, G., Kahr, M., Leitner, M., 2017. Determining optimal locations for charging stations of electric car-sharing systems under stochastic demand. Transportation Research Part B: Methodological 104, 17-35. http://dx.doi.org/10.1016/j.trb.2017.06.009.

Brendel, A. B., Lichtenberg, S., Brauer, B., Nastjuk, I., Kolbe, L. M., 2018. Improving electric vehicle utilization in carsharing: A framework and simulation of an e-carsharing vehicle utilization management system. Transportation Research Part D: Transport and Environment 64, $230-245$. http://dx.doi.org/10.1016/j.trd.2018.01.024.

Cepolina, E. M., Farina, A., 2014. A methodology for planning a new urban car sharing system with fully automated personal vehicles. European Transport Research Review 6 (2), 191-204. http://dx.doi.org/10.1007/s12544-013-0118-9.

Correia, G. H. A., Antunes, A. P., 2012. Optimization approach to depot location and trip selection in one-way carsharing systems. Transportation Research Part E: Logistics and Transportation Review 48 (1), 233 - 247. http://dx.doi.org/10.1016/j.tre.2011.06.003.

Costain, C., Ardron, C., Habib, K. N., 2012. Synopsis of users' behaviour of a carsharing program: A case study in Toronto. Transportation Research Part A: Policy and Practice 46 (3), 421-434. http://dx.doi.org/10.1016/j.tra.2011.11.005.

Crane, K., Ecola, L., Hassell, S., Natarah, S., 2012. An alternative approach for identifying opportunities to reduce emissions of greenhouse gases. Tech. rep., RAND Corporation. http://www.rand.org/content/dam/rand/pubs/technical_reports/2012/RAND_TR1170.pdf

De Lorimier, A., El-Geneidy, A. M., 2013. Understanding the factors affecting vehicle usage and availability in carsharing networks: A case study of Communauto carsharing system from Montréal, Canada. International Journal of Sustainable Transportation 7 (1), 35-51. http://dx.doi.org/10.1080/15568318.2012.660104.

Di Febbraro, A., Sacco, N., Saeednia, M., 2012. One-way carsharing: solving the relocation problem. Transportation research record 2319 (1), 113-120. http://dx.doi.org/10.3141/2319-13.

Di Febbraro, A., Sacco, N., Saeednia, M., 2019. One-way car-sharing profit maximization by means of user-based vehicle relocation. IEEE Transactions on Intelligent Transportation Systems 20 (2), 628-641. http://dx.doi.org/10.1109/TITS.2018.2824119.

Duncan, M., 2011. The cost saving potential of carsharing in a US context. Transportation 38 (2), 363-382. http://dx.doi.org/10.1007/s11116-0109304-y.

Firnkorn, J., Müller, M., 2011. What will be the environmental effects of new free-floating car-sharing systems? The case of Car2Go in Ulm. Ecological Economics 70 (8), 1519-1528. http://dx.doi.org/10.1016/j.ecolecon.2011.03.014. 
Fricker, C., Gast, N., 2016. Incentives and redistribution in homogeneous bike-sharing systems with stations of finite capacity. Euro journal on transportation and logistics 5 (3), 261-291. http://dx.doi.org/10.1007/s13676-014-0053-5.

Gambella, C., Malaguti, E., Masini, F., Vigo, D., 2018. Optimizing relocation operations in electric car-sharing. Omega 81,234 - 245. http://dx.doi.org/10.1016/j.omega.2017.11.007.

Illgen, S., Höck, M., 2018. Electric vehicles in car sharing networks - challenges and simulation model analysis. Transportation Research Part D: Transport and Environment 63, 377-387. http://dx.doi.org/10.1016/j.trd.2018.06.011.

Jorge, D., Correia, G. H. A., Barnhart, C., 2013. Comparing optimal relocation operations with simulated relocation policies in one-way carsharing systems. In: Transportation Research Board 92nd Annual Meeting. 13-4559. http://trid.trb.org/view.aspx?id=1242675

Jorge, D., Molnar, G., de Almeida Correia, G. H., 2015. Trip pricing of one-way station-based carsharing networks with zone and time of day price variations. Transportation Research Part B: Methodological 81 (2), 461-482. http://dx.doi.org/10.1016/j.trb.2015.06.003.

Kang, J., Hwang, K., Park, S., 2016. Finding factors that influence carsharing usage: Case study in Seoul. Sustainability 8 (8), 709. http://dx.doi.org/10.3390/su8080709.

Kaspi, M., Raviv, T., Tzur, M., 2014. Parking reservation policies in one-way vehicle sharing systems. Transportation Research Part B: Methodological 62, 35-50. http://dx.doi.org/10.1016/j.trb.2014.01.006

Kaspi, M., Raviv, T., Tzur, M., Galili, H., 2016. Regulating vehicle sharing systems through parking reservation policies: Analysis and performance bounds. European Journal of Operational Research 251 (3), 969-987. http://dx.doi.org/10.1016/j.ejor.2015.12.015.

Katzev, R., 2003. Car sharing: A new approach to urban transportation problems. Analyses of Social Issues and Public Policy 3 (1), 65-86. http://dx.doi.org/10.1111/j.1530-2415.2003.00015.x.

Kek, A. G. H., Cheu, R. L., Chor, M. L., 2006. Relocation simulation model for multiple-station shared-use vehicle systems. Transportation Research Record: Journal of the Transportation Research Board 1986, 81 - 88. http://dx.doi.org/10.3141/1986-13.

Kek, A. G. H., Cheu, R. L., Meng, Q., Fung, C. H., 2009. A decision support system for vehicle relocation operations in carsharing systems. Transportation Research Part E: Logistics and Transportation Review 45 (1), 149-158. http://dx.doi.org/10.1016/j.tre.2008.02.008.

Laporte, G., Meunier, F., Calvo, R. W., 2018. Shared mobility systems: an updated survey. Annals of Operations Research 271 (1), $105-126$. http://dx.doi.org/10.1007/s10479-018-3076-8.

Litman, T., 2000. Evaluating carsharing benefits. Transportation Research Record: Journal of the Transportation Research Board (1702), 31-35. http://dx.doi.org/10.3141/1702-04.

Ma, W., Wang, L., Li, L., 2018. Vehicle relocation triggering thresholds determination in electric carsharing system under stochastic demand. Journal of Advanced Transportation 2018, 15. http://dx.doi.org/10.1155/2018/3486741.

Martin, E. W., Shaheen, S. A., 2011. Greenhouse gas emission impacts of carsharing in north america. IEEE Transactions on intelligent transportation systems 12 (4), 1074-1086. http://dx.doi.org/10.1109/TITS.2011.2158539.

Morency, C., Trépanier, M., Martin, B., 2008. Object-oriented analysis of carsharing system. Transportation Research Record 2063 (1), $105-112$. http://dx.doi.org/10.3141/2063-13.

Nair, R., Miller-Hooks, E., 2011. Fleet management for vehicle sharing operations. Transportation Science 45 (4), 524-540. http://dx.doi.org/10.1287/trsc. 1100.0347 .

Nourinejad, M., Roorda, M. J., 2014. A dynamic carsharing decision support system. Transportation Research Part E: Logistics and Transportation Review 66, 36-50. http://dx.doi.org/10.1016/j.tre.2014.03.003.

Nourinejad, M., Zhu, S., Bahrami, S., Roorda, M. J., 2015. Vehicle relocation and staff rebalancing in one-way carsharing systems. Transportation Research Part E: Logistics and Transportation Review 81, 98-113. http://dx.doi.org/10.1016/j.tre.2015.06.012.

Repoux, M., Boyac1, B., Geroliminis, N., 2015. Simulation and optimization of one-way car-sharing systems with variant relocation policies. In: 94th Annual Meeting of the Transportation Research Board. No. 15-1907. Washington D.C., p. 18. http://trid.trb.org/view.aspx?id= 1337340

Schmöller, S., Weikl, S., Müller, J., Bogenberger, K., 2015. Empirical analysis of free-floating carsharing usage: The Munich and Berlin case. Transportation Research Part C: Emerging Technologies 56, 34-51. http://dx.doi.org/10.1016/j.trc.2015.03.008.

Schuster, T., Byrne, J., Corbett, J., Schreuder, Y., 2005. Assessing the potential extent of carsharing: A new method and its implications. Transportation Research Record: Journal of the Transportation Research Board (1927), 174-181. http://dx.doi.org/10.1177/0361198105192700120.

Shaheen, S., Cohen, A., Jaffee, M., 2018. Innovative mobility: Carsharing outlook. Tech. rep. https://escholarship.org/uc/item/1mw8n13h

Shaheen, S. A., Cohen, A. P., 2013. Carsharing and personal vehicle services: Worldwide market developments and emerging trends. International Journal of Sustainable Transportation 7 (1), 5-34. http://dx.doi.org/10.1080/15568318.2012.660103.

Song, T., Murata, T., 2018. Vehicle relocation scheduling method for car sharing service system based on Markov chain and genetic algorithm. In: Proceedings of the International MultiConference of Engineers and Computer Scientists. Vol. 2. http://www.iaeng.org/publication/ IMECS2018/IMECS2018_pp985-988.pdf

Wang, L., Liu, Q., Ma, W., 2019. Optimization of dynamic relocation operations for one-way electric carsharing systems. Transportation Research Part C: Emerging Technologies 101, 55-69. http://dx.doi.org/10.1016/j.trc.2019.01.005.

Waserhole, A., Jost, V., 2016. Pricing in vehicle sharing systems: Optimization in queuing networks with product forms. EURO Journal on Transportation and Logistics 5 (3), 293-320. http://dx.doi.org/10.1007/s13676-014-0054-4.

Waserhole, A., Jost, V., Brauner, N., 2013. Pricing techniques for self regulation in vehicle sharing systems. Electronic Notes in Discrete Mathematics 41, 149-156. http://dx.doi.org/10.1016/j.endm.2013.05.087.

Weikl, S., Bogenberger, K., 2015. A practice-ready relocation model for free-floating carsharing systems with electric vehicles - mesoscopic approach and field trial results. Transportation Research Part C: Emerging Technologies 57, 206-223. http://dx.doi.org/10.1016/j.trc.2015.06.024. 


\section{Appendix A. Mathematical model for the approximated upper bound}

In this appendix, we describe in details the mathematical model used to derive the approximate upper bound introduced in 3.3. The primary objective of the model is to maximize the number of accepted requests, subject to flow conservation constraints of vehicles and relocators, capacity constraints, parking and vehicle reservation constraints. The model assumes in-advance knowledge of the customers' requests over the planning horizon, typically, a day. In particular, for each request the model is given its booking time, starting time and origin station as well as ending time and destination station. The model simultaneously decides upon all requests to be accepted along the day and the relocations to be performed. These conditions allow the model to reject some requests even if the corresponding resources are available at the booking times, given that this improves the overall performance of the system. Such option does not exist in reality, or in our simulation as exact information regarding the future requests is not available.

To be consistent with the simulation conditions presented in Section 4.3, the mathematical model is solved iteratively to replicate the effect of unbalanced demand in consecutive days. At each iteration (i.e. each day), we serve a different set of requests and we use the final number of vehicles at each station as the initial number of vehicles at the next iteration.

We next define the sets, indices, parameters and variables. Further on, we present the mathematical model with the definitions of the objective function and the constraints.

Sets and Indices

$i \in I:$ demand requests

$j$ and $l \in J$ : stations

$t \in T:$ time intervals

\section{Parameters}

shiftstart/shiftend : start/end time intervals of personnel working shift

book/start/end $(i):$ book/start/end time intervals of demand request $i$

origin/dest $(i):$ origin/destination stations of demand request $i$

$\operatorname{drv} / \operatorname{mov}(j, l):$ total duration of a relocation/move from station $j$ to $l$

$\mathrm{NP}$ : maximum number of relocation personnel working simultaneously

$n_{j}^{0}:$ number of vehicles assigned to station $j$ at the first time interval

$b_{j}^{0}:$ number of vehicles reserved in station $j$ at the first time interval $\left(b_{j}^{0}=0 \quad \forall j \in J\right)$

$C A P_{j}$ : capacity of station $j$

$H$ : planning horizon, i.e. last time interval

Variables

$z_{i}: 1$ if demand request $i$ is served; 0 otherwise

$n_{j}^{t}$ : number of available vehicles in station $j$ at the beginning of time interval $t$

$b_{j}^{t}$ : number of reserved vehicles in station $j$ at the beginning of time interval $t$

$m_{j}^{t}$ : number of personnel in station $j$ at the beginning of time interval $t$

$r_{j l}^{t}$ : number of vehicles relocated by personnel from station $j$ to $l$ starting at time interval $t$

$p_{j l}^{t}$ : number of personnel relocated without vehicle from station $j$ to $l$ starting at time interval $t$ 


$$
\begin{aligned}
& \max \sum_{i} z_{i} \\
& \text { s.t. } n_{j}^{t+1}=n_{j}^{t}-\sum_{\substack{i: \operatorname{book}(i)=t \\
\operatorname{origin}(i)=j}} z_{i}-\sum_{l} r_{j l}^{t}+\sum_{\substack{i: \text { end }(i)=t \\
\operatorname{dest}(i)=j}} z_{i}+\sum_{l} r_{l j}^{t-\operatorname{drv}(l, j)} \\
& b_{j}^{t+1}=b_{j}^{t}-\sum_{\substack{i: \operatorname{start}(i)=t \\
\operatorname{origin}(i)=j}} z_{i}+\sum_{\begin{array}{c}
i: \operatorname{book}(i)=t \\
\operatorname{origin}(i)=j
\end{array}} z_{i} \\
& m_{j}^{t+1}=m_{j}^{t}-\sum_{l}\left(p_{j l}^{t}+r_{j l}^{t}\right)+\sum_{l}\left(p_{l j}^{t-\operatorname{mov}(l, j)}+r_{l j}^{t-\operatorname{drv}(l, j)}\right) \\
& n_{j}^{t} \geq \sum_{\substack{i: \operatorname{book}(i)=t \\
\operatorname{origin}(i)=j}} z_{i}+\sum_{l} r_{j l}^{t} \\
& b_{j}^{t} \geq \sum_{\substack{i: \operatorname{start}(i) \geq t \\
\operatorname{book}(i)<t \\
\operatorname{origin}(i)=j}} z_{i} \\
& n_{j}^{t}+b_{j}^{t} \leq \mathrm{CAP}_{j}-\sum_{\substack{i: \operatorname{book}(i) \leq t \\
\text { end }(i) \geq t \\
\operatorname{dest}(i)=j}} z_{i} \\
& 0 \leq n_{j}^{H}-\sum_{\substack{i: \operatorname{book}(i)=H \\
\operatorname{origin}(i)=j}} z_{i}-\sum_{l} r_{j l}^{H}+\sum_{\begin{array}{c}
i: \text { end }(i)=H \\
\operatorname{dest}(i)=j
\end{array}} z_{i}+\sum_{l} r_{l j}^{H} \leq \mathrm{CAP}_{j} \\
& \sum_{j} m_{j}^{\text {shiftstart }}=N P \\
& \sum_{j} m_{j}^{\text {shiftend }}=N P \\
& z_{i} \in\{0,1\} ; n_{j}^{t} \geq 0, m_{j}^{t} \geq 0, r_{j l}^{t} \geq 0, p_{j l}^{t} \geq 0
\end{aligned}
$$

The objective function A.1 maximizes the number of demand requests served. Constraints A.2, A.3 and A.4 are flow conservation equations for available vehicles, reserved vehicles and relocation personnel respectively. Constraints A.5 ensure the vehicles are available and waiting at the station the time interval before they are booked for a demand request or taken for a relocation. Constraints A.6 define the number of reserved vehicles as a station during time interval t. Note that vehicles are allowed to be reserved and leave in the same interval to account for short vehicle booking times. Constraints A.7 postulate that the maximum number of vehicles at any station at any time cannot be more than the capacity of the station minus the number of vehicles headed to that station, hence the number of currently reserved spots. Constraints A.8 ensure the number of vehicles at each station at the end of the planning period is between zero and the capacity of the station. Constraints A.9 and A.10 sets the start and end time of the relocators' shift and limit the number of personnel assigned to relocation operations. Constraints A.11 set the domains of each variable: $z_{i}$ are binary variables, all other variables are continuous and non-negative.

Unlike the dynamic policies presented in this paper and contrary to the system regulations, the model does not consider that relocators have to book and block the resources ahead of pick-up and drop-off time. This corresponds to a relaxation of the rules enforced in the system and is coherent with the use of this model as an approximate upper bound. We point out that some constraints could be easily adapted to take into account relocators' reservation of resources. For instance, enforcing parking spot reservation at the destination from the point vehicles start being relocated can be 
implemented by replacing A.7 by the following set of constraints A.12:

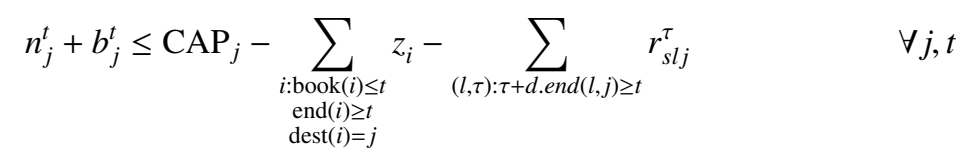

This model is adapted from the operations optimization model proposed by Boyac1 et al. (2017). The main differences between the approximation model used in this work and the framework of Boyac1 et al. (2017) are:

1. Relocation cost is not considered in the model. The main aim is to maximize the level of service.

2. In order to increase the utilization of the personnel, the constraints that require personnel to be present at the station during the entire time interval before the start time interval of the relocations are relaxed.

3. The number of relocation personnel is set to be constant.

4. As the studied system has a reasonable number of stations, no clustering is performed to reduce the size of the MILP.

5. Reservations at origin are introduced adding a new set of variables $b_{j}^{t}$ and sets of constraints. Other sets of constraints are modified to include these new variables and parking reservation requirements.

6. Charging level restrictions are disregarded and therefore it is assumed that vehicles are always charging level feasible.

7. The safety gap constraint that restricts the minimum time between consecutive demand requests served is relaxed. 